\title{
A Field Theory with Curvature and Anticurvature
}

\author{
M. I. Wanas ${ }^{1,2}$ and Mona M. Kamal ${ }^{2,3}$ \\ ${ }^{1}$ Astronomy Department, Faculty of Science, Cairo University, Giza 12613, Egypt \\ ${ }^{2}$ Egyptian Relativity Group (ERG), Cairo University, Giza 12613, Egypt \\ ${ }^{3}$ Mathematics Department, Faculty of Girls, Ain Shams University, Cairo 11566, Egypt \\ Correspondence should be addressed to M. I. Wanas; miwanas@sci.cu.edu.eg
}

Received 28 April 2014; Revised 9 August 2014; Accepted 10 August 2014; Published 14 September 2014

Academic Editor: Elias C. Vagenas

Copyright (C) 2014 M. I. Wanas and M. M. Kamal. This is an open access article distributed under the Creative Commons Attribution License, which permits unrestricted use, distribution, and reproduction in any medium, provided the original work is properly cited. The publication of this article was funded by SCOAP ${ }^{3}$.

\begin{abstract}
The present work is an attempt to construct a unified field theory in a space with curvature and anticurvature, the PAP-space. The theory is derived from an action principle and a Lagrangian density using a symmetric linear parameterized connection. Three different methods are used to explore physical contents of the theory obtained. Poisson's equations for both material and charge distributions are obtained, as special cases, from the field equations of the theory. The theory is a pure geometric one in the sense that material distribution, charge distribution, gravitational and electromagnetic potentials, and other physical quantities are defined in terms of pure geometric objects of the structure used. In the case of pure gravity in free space, the spherical symmetric solution of the field equations gives the Schwarzschild exterior field. The weak equivalence principle is respected only in the case of pure gravity in free space; otherwise it is violated.
\end{abstract}

\section{Motivations}

Historically, it is well known that, during the second decade of the past century, Einstein has solved some of the problems of gravity theory by introducing a new philosophy: "the geometrization philosophy." He has constructed a successful theory for gravity, the general relativity (GR). This philosophy and the quantization philosophy represent the two main philosophies, of the 20th century, in the domain of theoretical physics. Afterwards, it appeared that the geometrization philosophy is successful in the domain of macrophysics while microphysics is the domain of success of the quantization philosophy (the domain of short range forces).

Although GR is proved to be the best successful gravity theory in the domain of the solar and stellar systems, it has many problems in the domain of cosmological applications. The big bang model of the Universe suffers from such problems, for example, the initial singularity problem, particle horizons problem, and so on. Recently it is shown that GR cannot account for the results of observations, for example, SN type Ia, CMBR observations, the rotation curve of stars in spiral galaxies, and so forth, [1-3].
The need to modify GR, or to construct alternative theories, has increased in the past two or three decades. Some authors have suggested the use of the cosmological constant to solve such problems. But the cosmological constant itself has a big problem (cf. [4]). Another group has attempted to solve these problems in the context of Riemannian geometry by considering different functions of the curvature scalar $R, f(R)$ theories (cf. [5-7]). A third group has attempted to construct theories in the framework of geometries with nonvanishing torsion scalar $T$, the $f(T)$ theories (cf. [8-12]). Such scalars are usually used to construct the Lagrangian density of the theory suggested.

Authors almost use the absolute parallelism (AP) space as a space with nonvanishing torsion scalar $T$. It can be easily shown that, apart from a divergence term (cf. [13]), $T \equiv R$. So, both $T$ and $R$ can reproduce the same theory. For this reason the theory resulting from $T$ is known in the literature as the "teleparallel equivalent of general relativity" (TEGR). It is tempting to expect that both GR and TEGR give rise to the same physics. A main difference between these two theories is that the latter is written in the APspace which is much wider than the Riemannian one. Also, 
a difference between $f(R)$ and $f(T)$ theories is that the former gives fourth order differential equations while the latter gives second order differential equations for which it may be easier to find solutions.

In the present work, we draw the attention to the importance of the linear connection, of a certain space, in the physical contents of any geometric field theory, constructed in this space. We consider linear connection, of an appropriate geometry, as the most important geometric object when constructing geometric field theories. As an example, in the construction of GR, the role of Christoffel symbol is obvious in describing both field and motion. It is used in the definition of the curvature tensor (which represents the gravitational field) and in deriving the geodesic equation (describing motion in GR). So, one has to explore the role of any new linear connection, derived from the building blocks (BB) of the geometry used. In addition, torsion is defined as twice the skew part of the connection in geometries with nonsymmetric linear connections. So, in the context of geometrization philosophy, it is of importance to use any natural (here by natural we mean any geometric object defined in terms of the $\mathrm{BB}$ of the geometry used) modified linear nonsymmetric connection in constructing field theories. This may enable exploring the role of the above mentioned geometric objects in physics and predicting new physics, if any.

The use of a wider space with a general linear connection may give rise to new physics, which may be necessary to interpret recent observations. In the past two decades a wider type of geometry has been suggested and developed, the PAP-geometry [14-16]. Its linear connection is metric and nonsymmetric. This geometry has simultaneously nonvanishing torsion and curvature. Furthermore, a new geometric object called the "anticurvature" $[17,18]$ is defined which gives rise to "antigravity" [18]. The path equations (curves) of this geometry give rise to a repulsive force, together with the ordinary attractive force of gravity, when it is written in its linearized form [14]. Consequently, a theory written in the context of this geometry may help in solving some of the problems of GR especially the accelerating expansion of the Universe. Several successful applications have been done in the context of this geometry [18-21].

In the present paper we use the following terminology.

A geometry is a manifold $(M)$ equipped with building blocks (BB).

A space is a geometry admitting a linear connection.

A geometric structure is a space admitting a symmetry group (SG) or more.

The aim of the present work is to construct and analyze a field theory in the context of PAP-space and also to explore the role of the parameter of the PAP-space. To achieve this aim, the paper is arranged as follows: in Section 2, we give a brief account on the PAP-geometry and show that it can be reduced to Riemannian and conventional AP-spaces in some special cases. In Section 3, we suggest a certain Lagrangian density to construct a set of field equations using an action principle. In Section 4, we show how physics is extracted from the suggested geometric field theory before solving its field equations. The spherically symmetric case is studied in Section 5. Discussion of the results obtained and some concluding remarks are given in Section 6.

\section{The Underlying Geometry: PAP-Geometry}

The present work is carried out in the context of the "parameterized absolute parallelism-" (PAP-) geometry. This geometry has many advantages over both Riemannian and conventional AP geometries. In the present section, we give a brief review of this type of geometry. For more details readers are referred to [14-16].

"The PAP-geometry $\left(M, \lambda_{i}\right)$ is an $n$-dimensional differentiable manifold $M$ equipped with a set of $n$-linear independent vector fields $\lambda_{i}(x)(\mu(=1,2, \ldots, n)$ represents the vector component and $i(=1,2, \ldots, n)$ is used for the vector number. We use Greek indices for coordinate components written in a covariant or contravariant position. Latin indices are used to represent vector numbers, written in a lower position.)." These vector fields $\lambda_{i}$ are the same BB of conventional absolute parallelism- (AP-) geometry (cf. $[16,22-$ $24]$ ). As a consequence of this independence, the determinant $\lambda\left(=\left|\lambda_{i}\right|\right)$ is nonvanishing; that is, $\lambda \neq 0$. Now, we have the following (Einstein summation convention is taken over any repeated Greek index in the usual manner, while it is applied on repeated Latin indices wherever they appear.):

$$
\begin{aligned}
& { }_{i}^{\lambda^{\alpha}} \lambda_{\beta}=\delta_{\beta}^{\alpha}, \\
& { }_{i}^{\alpha} \lambda_{j}=\delta_{i j},
\end{aligned}
$$

where $\lambda^{\alpha}$ are the contravariant components of the vector fields $\lambda_{i}^{i}$, that is, the normalized cofactor of $\lambda_{i}$. One can define the following second order symmetric tensors:

$$
\begin{aligned}
& g_{\mu \nu} \stackrel{\text { def. }}{=} \lambda_{i} \mu \underset{i}{\lambda_{\nu},} \\
& g^{\mu \nu} \stackrel{\text { def. }}{=} \lambda_{i}^{\mu} \lambda_{i} \lambda^{\nu} .
\end{aligned}
$$

Now, since $\lambda$ is nonvanishing, it is easy to show that $g=$ $\left|g_{\mu \nu}\right| \neq 0$ and consequently we defined $g^{\mu \nu}$, the normalized cofactor of $g_{\mu \nu}$. Then we can write

$$
g^{\alpha \mu} g_{\alpha \nu}=\delta_{. v}^{\mu}
$$

So, (3) and (4) can be used as the metric tensor of a Riemannian space in the framework of the PAP-geometry.

2.1. PAP-Linear Connection. Motivated by some arguments $[14,15]$, the PAP-linear connection $\nabla_{. \mu \nu}^{\alpha}$ has been defined as

$$
\nabla_{\cdot \mu \nu}^{\alpha} \stackrel{\text { def. }}{=}\left\{\begin{array}{c}
\alpha \\
\mu \nu
\end{array}\right\}+\stackrel{*}{\gamma}_{\cdot \mu \nu}^{\alpha}
$$

where $\left\{\begin{array}{c}\alpha \\ \mu \nu\end{array}\right\}$ is the Christoffel symbol of the second kind defined, as usual, by

$$
\left\{\begin{array}{c}
\alpha \\
\mu \nu
\end{array}\right\} \stackrel{\text { def. }}{=} \frac{1}{2} g^{\alpha \varepsilon}\left(g_{\varepsilon \mu, \nu}+g_{\nu \varepsilon, \mu}-g_{\mu \nu, \varepsilon}\right) .
$$


And $\stackrel{*}{\gamma}_{. \mu \nu}^{\alpha}$ is a third order tensor, called the parameterized contortion, defined by $\stackrel{\gamma}{\gamma}_{. \mu \nu}^{\alpha} \stackrel{\text { def. }}{=} b \lambda_{i}^{\alpha} \lambda_{i} \lambda_{i, \nu}$ (Comma (,) is used for ordinary partial differentiation and (;) for covariant differentiation using Christoffel symbol.). Here $b$ is a dimensionless parameter, characterizing this type of geometry, to be discussed later. The space constructed using the parameterized linear connection (6) is called the PAPspace $(M, \underset{i}{\lambda}$, and $\nabla)$.

2.2. Tensor Derivatives. Since $\nabla_{. \mu \nu}^{\alpha}$ is a nonsymmetric linear connection, one can define the following tensor derivatives:

$$
\begin{gathered}
A_{. \| \beta}^{+} \stackrel{\text { def. }}{=} A_{, \beta}^{\alpha}+A^{\mu} \nabla_{\mu \beta}^{\alpha}, \\
A_{. \| \beta}^{\alpha} \stackrel{\text { def. }}{=} A_{, \beta}^{\alpha}+A^{\mu} \nabla_{\beta \mu}^{\alpha}, \\
A_{. \| \beta}^{\alpha} \stackrel{\text { def. }}{=} A_{, \beta}^{\alpha}+A^{\mu} \nabla_{(\mu \beta)}^{\alpha},
\end{gathered}
$$

where $A^{\alpha}$ is any arbitrary vector, and $\nabla_{.(\mu \nu)}^{\alpha}$ is the symmetric part of $\nabla_{. \mu \nu}^{\alpha}(6)$, defined as

$$
\begin{aligned}
\nabla_{.(\mu \nu)}^{\alpha} \stackrel{\text { def. }}{=} \frac{1}{2}\left(\nabla_{\cdot \mu \nu}^{\alpha}+\nabla_{\cdot \nu \mu}^{\alpha}\right) \\
=\left\{\begin{array}{c}
\alpha \\
\mu \nu
\end{array}\right\}+\frac{1}{2} \stackrel{\Delta}{*}_{\cdot \mu \nu}^{\alpha},
\end{aligned}
$$

where

$$
\stackrel{*}{\Delta}_{. \mu \nu}^{\alpha} \stackrel{\text { def. }}{=} \gamma_{\cdot \mu \nu}^{\alpha}+\stackrel{*}{\gamma}_{\cdot \nu \mu}^{\alpha} \text {. }
$$

It can be easily shown that (9) is a symmetric linear connection under the effect of group of general coordinate transformation.

2.3. The Parameterized Torsion Tensor. Since parameterized linear connection $\nabla_{. \mu \nu}^{\alpha}$ is nonsymmetric, as stated above, it has a skew-symmetric part $\stackrel{*}{\Lambda}_{, \mu \nu}^{\alpha}$ defined by

$$
\begin{aligned}
\stackrel{*}{\Lambda}_{\cdot \mu \nu}^{\alpha} & \stackrel{\text { def. }}{=} \nabla_{\cdot \mu \nu}^{\alpha}-\nabla_{\cdot \nu \mu}^{\alpha} \\
& ={ }^{*}{ }^{\alpha}{ }_{. \mu \nu}-{ }^{*}{ }_{. \nu \mu}^{\alpha}=b \Lambda_{. \mu \nu}^{\alpha},
\end{aligned}
$$

which is called the parameterized torsion tensor and $\Lambda_{. \mu \nu}^{\alpha}$ is the torsion tensor of the AP-space. The relation between the parameterized torsion and the parameterized contortion, is given by

$$
\stackrel{*}{\gamma}_{\mu \nu}^{\alpha}=\frac{1}{2}\left(\stackrel{*}{\Lambda}_{\mu \nu}^{\alpha}-\stackrel{*}{\Lambda}_{\mu, \nu}^{\alpha}-\stackrel{*}{\Lambda}_{\nu \cdot \mu}^{\alpha}\right) .
$$

\begin{tabular}{|c|c|}
\hline & \\
\hline $\begin{array}{l}\text { Skew tensors } \\
* \text { def. } *\end{array}$ & Symmetric tensors \\
\hline$\xi_{\mu \nu} \stackrel{\mu .}{=} \gamma_{\mu \nu, \| e}^{\epsilon}$ & \\
\hline$\stackrel{*}{\zeta_{\mu \nu}} \stackrel{\text { def. }}{=} \stackrel{*}{c}_{\alpha} \gamma_{\mu \nu}^{\alpha}$ & \\
\hline$\stackrel{H}{\eta}_{\mu \nu} \stackrel{\text { def. }}{=} c_{\alpha}^{*}{ }_{\alpha}^{*}{ }_{\mu \nu}^{\alpha}$ & $\stackrel{*}{\phi}_{\mu \nu} \stackrel{\text { def. }}{=} c_{\alpha}^{*} \stackrel{*}{\Delta}_{\mu \nu}^{\alpha}$ \\
\hline $\bar{\chi}_{\mu \nu}^{*} \stackrel{\text { def. }}{=} \Lambda_{\mu \nu \|_{e}}^{\epsilon}$ & $\dot{\psi}_{\mu \nu}^{*} \stackrel{\text { def. }}{=} \Delta_{, \mu \nu \| \epsilon}^{\epsilon}$ \\
\hline $\mathcal{E}_{\mu \nu}^{*} \stackrel{\text { def. }}{=} \mathcal{C}_{\mu \| \nu}^{*}-{\stackrel{*}{\mathcal{C}_{\nu \| \mu}}}^{*}$ & $\theta_{\mu \nu}^{*} \stackrel{\text { def. }}{=} \mathcal{c}_{\mu \| \nu}^{*}+\stackrel{*}{\mathcal{c}_{\nu \mid \mu}}$ \\
\hline 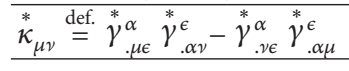 & 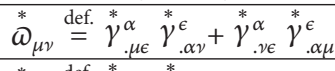 \\
\hline & 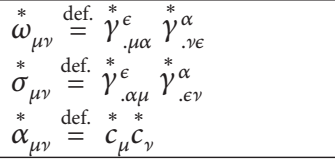 \\
\hline
\end{tabular}

The contraction of the parameterized torsion (11) or contortion (12) of the PAP-space gives the parameterized basic vector $\stackrel{*}{c}_{\mu}$, which is defined as

$$
\stackrel{*}{c_{\mu}} \stackrel{\text { def. }}{=} \stackrel{*}{\Lambda}_{\cdot \mu \alpha}^{\alpha}=\stackrel{*}{\gamma}_{\cdot \mu \alpha}^{\alpha} .
$$

TABLE 1: PAP second order world tensors [25].

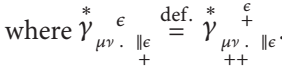

2.4. Second Order Tensors and Scalars. Table 1 [25] gives the definition of a list of second order tensors: These tensors are defined using the above parameterized third order tensors and the parameterized basic vector. The table is similar to that constructed by Mikhail in 1962 [22] in the context of APgeometry.

Using tensors given in Table 1, we can define many scalars

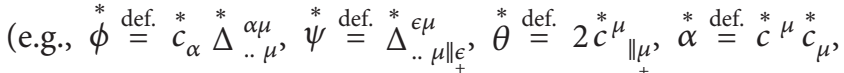
$\stackrel{*}{\omega} \stackrel{\text { def. }}{=} 2 \stackrel{*}{\gamma}_{. .}^{\alpha \mu} \stackrel{*}{\gamma}_{. \alpha \mu}^{\epsilon} \stackrel{*}{\omega} \stackrel{\text { def. }}{=} \stackrel{*}{\gamma}_{., \alpha}^{\epsilon} \stackrel{*}{\gamma}_{. \mu \epsilon}^{\alpha}$, and $\left.\stackrel{*}{\sigma} \stackrel{\text { def. }}{=} \stackrel{*}{\gamma}_{. \alpha \mu}^{\epsilon} \stackrel{*}{\gamma}_{\epsilon}^{\alpha, \mu}\right)$.

Among these tensors, we have a set of second order traceless tensors (e.g., $\left(\stackrel{*}{\phi}_{\mu \nu}+2 \stackrel{*}{\alpha}_{\mu \nu}\right),\left(\stackrel{*}{\psi}_{\mu \nu}+\stackrel{*}{\theta}_{\mu \nu}\right)$, and $\left(\stackrel{*}{\oplus}_{\mu \nu}+\right.$ $\left.\left.2 \stackrel{*}{\omega}_{\mu \nu}\right)\right)$ with possible linear combination. Such objects may be of physical interest.

2.5. Parameterized Curvature and Anticurvature Tensors. We can define many parameterized curvature tensors in the context of the PAP-space, none of which are vanishing. Here we are interested in the curvature corresponding to the linear connection (9). So, the definition of this parameterized curvature tensor may be written as

$$
B_{\cdot \mu \nu \sigma}^{\alpha} \stackrel{\text { def. }}{=} \nabla_{.(\mu \sigma), \nu}^{\alpha}-\nabla_{.(\mu \nu), \sigma}^{\alpha}+\nabla_{.(\mu \sigma)}^{\epsilon} \nabla_{.(\epsilon \nu)}^{\alpha}-\nabla_{.(\mu \nu)}^{\epsilon} \nabla_{.(\epsilon \sigma)}^{\alpha} .
$$

Substituting from (9) into (14), the parameterized curvature (14) can be split in two parts as follows:

$$
B_{. \mu \nu \sigma}^{\alpha}=R_{. \mu \nu \sigma}^{\alpha}+\stackrel{*}{Q}_{. \mu \nu \sigma}^{\alpha}
$$

where

$$
R_{\cdot \mu \nu \sigma}^{\alpha} \stackrel{\text { def. }}{=}\left\{\begin{array}{c}
\alpha \\
\mu \sigma
\end{array}\right\}_{, \nu}-\left\{\begin{array}{c}
\alpha \\
\mu \nu
\end{array}\right\}_{, \sigma}+\left\{\begin{array}{c}
\alpha \\
\epsilon \nu
\end{array}\right\}\left\{\begin{array}{c}
\epsilon \\
\mu \sigma
\end{array}\right\}-\left\{\begin{array}{c}
\alpha \\
\epsilon \sigma
\end{array}\right\}\left\{\begin{array}{c}
\epsilon \\
\mu \nu
\end{array}\right\}
$$


is the conventional Riemann-Christoffel curvature tensor and $\stackrel{*}{Q}^{\alpha}{ }_{\mu \nu \sigma}$ is the parameterized anticurvature defined by

$$
\begin{aligned}
& \stackrel{*}{Q}_{. \mu \nu \sigma}^{\alpha} \stackrel{\text { def. }}{=} \frac{1}{2}\left(\stackrel{*}{\Delta}_{. \mu \sigma . \|{ }_{+}^{\alpha}}^{\alpha}-\stackrel{*}{\Delta}_{. \mu \nu . \|{ }_{+}^{\alpha}}^{\alpha}+\stackrel{*}{\Lambda}_{. \sigma \nu}^{\epsilon} \stackrel{*}{\Delta}_{. \mu \epsilon}^{\alpha}\right. \\
& -\frac{3}{2} \stackrel{*}{\gamma}_{. \mu \sigma}^{\epsilon} \stackrel{*}{\gamma}_{. \epsilon \nu}^{\alpha}+\frac{3}{2} \stackrel{*}{\gamma}_{. \epsilon \sigma}^{\alpha} \stackrel{*}{\gamma}_{\cdot \mu \nu}^{\epsilon} \\
& +\frac{1}{2} \stackrel{*}{\Lambda}_{. \nu \epsilon}^{\alpha} \stackrel{*}{\gamma}_{. \sigma \mu}^{\epsilon}+\frac{1}{2} \stackrel{\gamma}{\gamma}_{. \sigma \epsilon}^{*} \stackrel{*}{\Lambda}_{. \mu \nu}^{\epsilon} \\
& \left.-\frac{1}{2} \stackrel{*}{\gamma}_{. \nu \epsilon}^{\alpha} \stackrel{*}{\gamma}_{. \mu \sigma}^{\epsilon}+\frac{1}{2} \stackrel{*}{\gamma}_{. \epsilon \sigma}^{\alpha} \stackrel{*}{*}_{. \nu \mu}^{\epsilon}\right)
\end{aligned}
$$

which is a tensor of type $(1,3)$. Note that the first term on the right-hand side of (15) is independent of the parameter $b$. This is an important property that is discussed later. It is to be considered that the parameterized curvature (14) is, in general, a nonvanishing tensor. Equation (15) shows that it is split into two parts. The first (16) depends only on Christoffel symbol of the second kind, which coincides with Riemannian-Christoffel curvature tensor of the Riemannian space. The second (17) depends only on the contortion (or torsion via (11)) of the PAP-space. For certain properties, the tensor (17) is called the parameterized anticurvature tensor $[17,18,26]$.

The contracted curvature tensor (by equating $\alpha$ and $\sigma$ in (14)) can be written in the following form:

$$
\begin{aligned}
& B_{\mu \nu} \stackrel{\text { def. }}{=} R_{\mu \nu}+\frac{1}{2} c_{\mu\|\|_{+}}^{*}-\frac{1}{2} \stackrel{*}{\Delta}_{. \mu \nu \cdot \|_{+}^{\alpha}}^{\alpha}+\frac{1}{4} \stackrel{*}{\gamma}_{. \alpha \nu}^{\epsilon} \stackrel{*}{\Lambda}_{. \epsilon \mu}^{\alpha} \\
& -\frac{3}{4} \stackrel{*}{\gamma}_{. \nu \alpha}^{\epsilon} \stackrel{*}{\gamma}_{. \mu \epsilon}^{\alpha}-\frac{1}{4} \stackrel{*}{\gamma}_{. \nu \alpha}^{\epsilon} \stackrel{*}{\gamma}_{. \epsilon \mu}^{\alpha} \\
& +\frac{3}{4} \stackrel{*}{\epsilon}_{\epsilon} \stackrel{*}{\gamma}_{\cdot \mu \nu}^{\epsilon}+\frac{1}{4} \stackrel{*}{\epsilon}_{\epsilon} \stackrel{*}{\gamma}_{\cdot \nu \mu}^{\epsilon}
\end{aligned}
$$

Also, the parameterized scalar curvature can be defined by contracting (18), which gives

$$
\begin{aligned}
B \stackrel{\text { def. }}{=} g^{\mu \nu} B_{\mu \nu}= & R+\frac{3}{2} \stackrel{*}{c}_{\cdot \|_{+}^{\mu}}^{\mu}-\stackrel{*}{c}_{\mu} \stackrel{*}{c} \mu^{\mu}-\frac{1}{4} \stackrel{*}{\Lambda}^{\alpha \mu \nu}{ }_{\Lambda}^{*} \Lambda_{\alpha \mu \nu} \\
& +\frac{1}{4} \stackrel{*}{\Lambda}^{\alpha \mu \nu}{ }_{\Lambda}^{*}{ }_{\nu \alpha \mu},
\end{aligned}
$$

where

$$
\stackrel{*}{\Lambda} \stackrel{\alpha \nu \nu}{\stackrel{\text { def. }}{=}} g^{\beta \mu} g^{\gamma \nu} \Lambda_{. \beta \gamma}^{*}, \quad \stackrel{*}{\Lambda}{ }_{\alpha \mu \nu} \stackrel{\text { def. }}{=} g_{\alpha \beta} \stackrel{*}{\Lambda}_{. \mu \nu}^{\beta} .
$$

2.6. Path Equations. A general path equation for this type of space has been derived [14] and can be written in the following form:

$$
\frac{d^{2} x^{\mu}}{d \tau^{2}}+\left\{\begin{array}{c}
\mu \\
\alpha \beta
\end{array}\right\} \frac{d x^{\alpha}}{d \tau} \frac{d x^{\beta}}{d \tau}=-\Lambda_{(\alpha \beta)}^{*} \frac{d x^{\alpha}}{d \tau} \frac{d x^{\beta}}{d \tau},
$$

where $\tau$ is the parameter characterizing this path, and $d x^{\alpha} / d \tau$ is the tangent of the path.

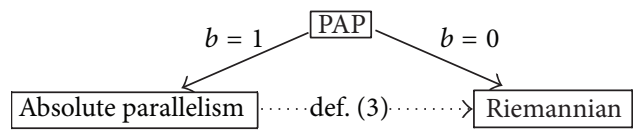

FIGURE 1: AP and Riemannian spaces as special cases of the PAPgeometry.

2.7. Special Cases. It is well known that for any AP-space there exists an associated Riemannian one, with metric tensor defined by (3). It is to be considered that Riemannian geometry is not a special case of the AP-space (cf. [22]). In PAP-space the situation is different. It can be easily shown that Riemannian space and the AP-space are just special cases of the PAP-space, corresponding to $b=0$ and $b=1$, respectively. All features of the Riemannian geometry can be covered, by taking $b=0$, in (6). Also, the PAP-space reduces to the AP-space if we take $b=1$. So, Riemannian geometry is not associated with the PAP-geometry, but it is obtained as a special case. This important features facilitate comparison between the results obtained in the context of the PAPgeometry and those obtained in the context of Riemannian or AP geometries.

Figure 1 shows the relations between PAP-, AP-, and Riemannian spaces. Solid arrows give the direction for some special cases, while the dashed one gives the association case. It is to be considered that there is a wide range of spaces (a discrete spectra) between the above mentioned two special cases (i.e., between $b=0$ and $b=1$ ). This shows how wide the PAP-space is compared with Riemannian or conventional AP-spaces.

\section{The Equations of the Theory}

3.1. An Action Principle: Field Equations. In this section we derive a set of field equations corresponding to the scalar curvature (19) using an action principle. The Lagrangian density corresponding to the scalar (19) is defined by

$$
\mathscr{L} \stackrel{\text { def. }}{=} \lambda B
$$

which can be written, using (19), as

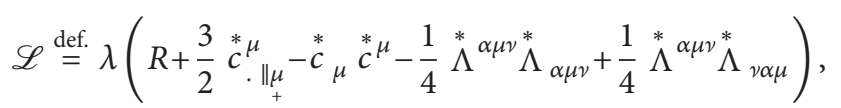

or equivalently, it can be written as

$$
\begin{gathered}
\mathscr{L} \stackrel{\text { def. }}{=} \lambda\left(R+\frac{3}{2} \stackrel{*}{c}_{\cdot ; \mu}^{\mu}+\frac{1}{2} \stackrel{*}{c}_{\mu}{ }^{{ }^{*}}{ }^{\mu}-\frac{1}{4} \stackrel{*}{\Lambda}^{\alpha \mu \nu}{ }^{*} \Lambda_{\alpha \mu \nu}\right. \\
\left.+\frac{1}{4} \stackrel{*}{\Lambda}^{\alpha \mu \nu}{ }^{*} \Lambda_{\nu \alpha \mu}\right) .
\end{gathered}
$$

Since, as it is well known, the term $\left(\lambda \stackrel{*}{\mathcal{c}}{ }^{\mu}\right)_{, \mu}$ has no contribution to the variation; we write (24) in the following form;

$$
\mathscr{L}=\lambda\left(R+\frac{1}{2} \stackrel{*}{c}_{\mu} \stackrel{*}{c}^{\mu}-\frac{1}{4} \stackrel{*}{\Lambda}^{\alpha \mu \nu}{ }_{\Lambda}^{*}{ }_{\alpha \mu \nu}+\frac{1}{4} \stackrel{*}{\Lambda}^{\alpha \mu \nu}{ }^{*} \Lambda_{\nu \alpha \mu}\right) .
$$


The Euler-Lagrange equation for the function $\mathscr{L}$ is

$$
\frac{\delta \mathscr{L}}{\delta \lambda_{i}} \stackrel{\text { def. }}{=} \frac{\partial \mathscr{L}}{\partial \lambda_{i}}-\frac{\partial}{\partial x^{\mu}} \frac{\partial \mathscr{L}}{\partial \lambda_{i}, \mu}=0 .
$$

For evaluating (26), we can define

$$
\underset{i}{K} \stackrel{\text { def. }}{=} \frac{\delta \mathscr{L}}{\delta \lambda_{\beta}}=0 .
$$

Multiplying both sides by $\lambda_{i}$ we obtain, after necessary manipulations,

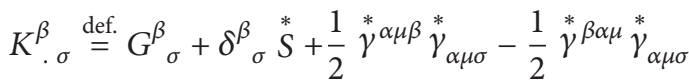

$$
\begin{aligned}
& +\frac{1}{2} b \stackrel{*}{\gamma}^{\alpha \mu \beta} \stackrel{*}{\gamma}_{\sigma \alpha \mu}+b \stackrel{*}{\gamma} \beta \alpha \mu^{*} \gamma_{\alpha \mu \sigma}+\frac{3}{2} b \stackrel{*}{\gamma} \beta \alpha \mu^{*} \gamma_{\sigma \mu \alpha} \\
& -\stackrel{*}{c}^{\mu} \stackrel{*}{\gamma}_{\cdot \mu \sigma}^{\beta}+\frac{3}{2} b \stackrel{*}{c}^{\mu} \stackrel{*}{\gamma}_{\cdot \mu \sigma}^{\beta}-\frac{1}{2} b \stackrel{*}{c}^{\mu} \stackrel{*}{\gamma}_{. \sigma \mu}^{\beta}
\end{aligned}
$$

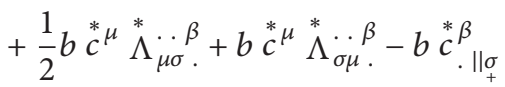

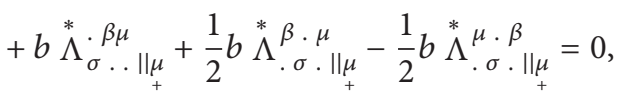

where

$$
\begin{gathered}
G_{\sigma}^{\beta} \stackrel{\text { def. }}{=} R_{\sigma}^{\beta}-\frac{1}{2} \delta_{\sigma}^{\beta} R, \\
\stackrel{*}{\text { def. }}=\frac{b}{2} \stackrel{*}{\theta}-\frac{1}{4}(1-2 b) \stackrel{*}{\phi}+\frac{1}{4} \stackrel{*}{\sigma}-\frac{1}{4} \stackrel{*}{\omega},
\end{gathered}
$$

and any scalar $E$ of a second order symmetric tensor $E_{\mu \nu}$ given in Table 1 is defined, as usual by $E \stackrel{\text { def. }}{=} g^{\mu \nu} E_{\mu \nu}$. Using Table 1, the field equations (28) may be written in the following form:

$$
\begin{aligned}
K_{\mu \nu} \stackrel{\text { def. }}{=} & G_{\mu \nu}+g_{\mu \nu} \stackrel{*}{S}-\frac{1}{2} \stackrel{*}{\sigma}_{\mu \nu}+\left(-\frac{1}{4}+\frac{3 b}{4}\right) \stackrel{*}{\omega}_{\mu \nu}+\frac{3}{2} b \stackrel{*}{\omega}_{\mu \nu} \\
& +\left(\frac{1}{2}-\frac{5 b}{4}\right) \stackrel{*}{\phi}_{\mu \nu}-\frac{1}{2} b \stackrel{*}{\theta}_{\mu \nu}+\frac{3}{4} b \stackrel{*}{\psi}_{\mu \nu}+\left(-\frac{1}{4}+\frac{b}{4}\right) \stackrel{*}{\kappa}_{\mu \nu} \\
& +\left(\frac{1}{2}-\frac{3 b}{4}\right) \stackrel{*}{\eta}_{\mu \nu}+\frac{1}{2} b \stackrel{*}{\zeta}_{\mu \nu}-\frac{1}{2} b \mathcal{\varepsilon}_{\mu \nu} \\
& +\frac{1}{4} b \chi_{\mu \nu}^{*}-\frac{1}{2} b \stackrel{*}{\xi}_{\mu \nu}=0 .
\end{aligned}
$$

These are the field equations of the suggested theory corresponding to the Lagrangian density (25). In general, $K_{\mu \nu}$ is nonsymmetric, and (30) is generally covariant and made of pure geometric objects defined in terms of the $\mathrm{BB}$ of the PAP-geometry. In four dimensions (30) represents 16 field equations in the 16 unknown functions $\lambda_{i}$ (the BB of the PAPgeometry).
3.2. The Equations of Motion and Physical Meaning of b. For the present theory, and in the framework of the geometrization of physics, we use the path equation (21) as an equation of motion. This equation can be used to describe the motion of a spinning test particle in the gravitational field given by the theory. In the case of motion of a charged spinning particle a modified version of (21) is to be used.

For new theories, the physical meaning for a geometric object, constant or parameter, is obtained with more ease using the equations of motion rather than using the field equations. Afterwards, this meaning is to be used for interpreting solutions of the field equations. For example, in the case of GR, the attribution of physical meaning to the metric tensor has been obtained by comparing the linearized geodesic equation with Newtonian motion. A similar procedure has been followed to get the physical meaning of $b$ in the present theory as follows.

(i) In the context of geometrization philosophy, the path equations (21) have been used to represent trajectories of spinning test particles in a gravitational field [14].

(ii) For several reasons [15] the parameter $b$ is suggested to take the form

$$
b \stackrel{\text { def. }}{=} \frac{N}{2} \alpha \gamma,
$$

where $N$ is a natural number taking the values $0,1,2, \ldots$ for particles with spin $0,1 / 2,1, \ldots$, respectively; $\alpha$ is fine structure constant and $\gamma$ is a free parameter depending on the system under consideration. In general $b$ is not a constant but a parameter depending on the spin of the moving particle and the system under consideration (the source of the gravitational field).

(iii) The term on the right-hand side of $(21)-b \Lambda_{(\alpha \beta)}^{\mu}\left(d x^{\alpha} /\right.$ $d \tau)\left(d x^{\beta} / d \tau\right)$ has been interpreted as an interaction between the spin of the moving particle and the torsion of the background field. It is called "spin-torsion interaction" term [14], with $b$ being the coupling of this interaction [20].

Two conditions for this interaction to disappear are either using geometries with vanishing torsion (the case of Riemannian geometry) or the moving particle being spinless.

(iv) Concerning the experimental results confirming the value and physical meaning of the parameter $b$ we caught here two experiments.

(a) The COW-experiment: this experiment, carried out many times by Colella, Overhauser, and Wener [27-29] concerns quantum interference of thermal neutrons under the influence of the Earth's gravitational field. The well known discrepancy of this experiment has been successfully interpreted [19] using the spin torsion interaction of (21) with $2 \geq \gamma \geq 1$ for the Earth's system. 
(b) The gravitomagnetism experiment: this experiment is also carried out in the Earth's gravitational field using atomic clocks. The results of this experiment have been used to fix a value for the parameter $b$ [20]. The results obtained confirmed that $\gamma$ is of order unity for the Earth's system.

(v) It has been shown that $b$ reflects the existence of some quantum features for any nonsymmetric geometry $[30,31]$ (a geometry with nonvanishing torsion, that is, with nonsymmetric linear connection).

\section{Extraction of Physics}

The field equations of the theory (30) are pure geometric equations. In order to explore the physics covered by the suggested theory we are going to use three methods. In the first method, physics is gained by comparing the present theory with nonlinear field theories. More physics is obtained by using the second method, that is, comparing the theory with linear field theories. The third method is used to explore the capabilities of a geometric structure, used for applications, for representing physical fields. All three methods are used before solving the field equations of the theory.

4.1. Analysis of the Field Equations. For this analysis, we split the field equations into two parts. The symmetric part is to be compared with GR, while the skew symmetric part is to be compared with the electromagnetic sector of EinsteinMaxwell theory.

4.1.1. The Symmetric Part of the Field Equations. The symmetric part of the field equations (30) is

$$
K_{(\mu \nu)} \stackrel{\text { def. }}{=} \frac{1}{2}\left(K_{\mu \nu}+K_{\nu \mu}\right)=0 \text {. }
$$

Using (30), the above equations may be written in the form

$$
R_{\mu \nu}-\frac{1}{2} g_{\mu \nu} R=\stackrel{*}{T}_{\mu \nu}
$$

where the left-hand side is Einstein tensor and right-hand side is a second order pure geometric tensor defined by

$$
\begin{aligned}
& \stackrel{*}{T}_{\mu \nu} \stackrel{\text { def. }}{=}-g_{\mu \nu} \stackrel{*}{S}^{1}+\frac{1}{2} \stackrel{*}{\sigma}_{\mu \nu}+\left(\frac{1}{4}-\frac{3 b}{4}\right) \stackrel{*}{\omega}_{\mu \nu}-\frac{3}{2} b \stackrel{*}{\omega}_{\mu \nu} \\
& -\left(\frac{1}{2}-\frac{5 b}{4}\right) \stackrel{*}{\phi}_{\mu \nu}+\frac{1}{2} b{ }^{*} \theta_{\mu \nu}-\frac{3}{4} b \stackrel{*}{\psi}_{\mu \nu} .
\end{aligned}
$$

All second order tensors written on the right-hand side of (34) are defined in Table 1.

Now to gain some physical information from the field equations of the theory, we compare the symmetric part of the field equations (33) with Einstein field equations of GR, namely,

$$
R_{\mu \nu}-\frac{1}{2} g_{\mu \nu} R=-\kappa T_{\mu \nu}
$$

We may attribute the following physical meaning, as a consequence of this comparison, to the symmetric tensors of (33).

(1) The tensor $g_{\mu \nu}$ can be considered as representing the gravitational potential in the suggested theory.

(2) The left-hand side of (33) represents the status and evaluation of a gravitational field whose potential is given by $g_{\mu \nu}$ as stated above.

(3) The tensor $\stackrel{*}{T}_{\mu \nu}$ may be used as a pure geometric representation of a material-energy distribution generating the field. Justifications of this suggestion are as follows.

(a) It is a second order symmetric tensor.

(b) It can be considered as a source of the gravitational field appearing on the left-hand side of (33).

(c) The left-hand side of (33) satisfies Bianchi differential identity, and consequently the tensor $\stackrel{*}{T}_{\mu \nu}$ will satisfy a generalized conservation law

$$
\stackrel{*}{T}^{\mu \nu}{ }_{; \nu}=0 \text {. }
$$

Further justifications are given in the next subsections.

4.1.2. The Skew Part of the Field Equations. This part is given by

$$
K_{[\mu \nu]} \stackrel{\text { def. }}{=} \frac{1}{2}\left(K_{\mu \nu}-K_{\nu \mu}\right)=0
$$

Using (30), (37), the above equation may be written in the form

$$
\stackrel{*}{F}_{\mu \nu}=-\frac{1}{2} b\left(\stackrel{*}{c}_{\mu, \nu}-\stackrel{*}{c}_{\nu, \mu}\right),
$$

where the right-hand side is a curl of the vector $\stackrel{*}{c}_{\mu}$ and the left-hand side is a second order, pure geometric, skew tensor defined by

$$
\begin{aligned}
\stackrel{*}{F}_{\mu \nu} \stackrel{\text { def. }}{=} & \left(\frac{1}{4}-\frac{b}{4}\right) \stackrel{*}{\kappa}_{\mu \nu}-\left(\frac{1}{2}-\frac{b}{4}\right) \stackrel{*}{\eta}_{\mu \nu}-\frac{1}{2} b \zeta_{\mu \nu}^{*}-\frac{1}{4} b \chi_{\mu \nu}^{*} \\
& +\frac{1}{2} b \xi_{\mu \nu}^{*},
\end{aligned}
$$

and all second order skew tensors, written on the right-hand side of (39), are defined in Table 1. Also, using equation (38) we can show that

$$
\stackrel{*}{F}_{\mu \nu \| \alpha}+\stackrel{*}{F}_{\alpha \mu \| \nu}+\stackrel{*}{F}_{\nu \alpha \| \mu} \equiv \stackrel{*}{F}_{\mu \nu, \alpha}+\stackrel{*}{F}_{\alpha \mu, \nu}+\stackrel{*}{F}_{\nu \alpha, \mu}=0 .
$$

Using the skew tensor $\stackrel{*}{F}_{\mu \nu}$, we can define the following tensor density:

$$
\stackrel{*}{\mathfrak{F}}^{\mu \nu} \stackrel{\text { def. }}{=} \lambda \stackrel{*}{F}^{\mu \nu}
$$


from which we define the vector density

$$
\stackrel{*}{\mathfrak{J}}^{\mu} \stackrel{\text { def. }}{=} \stackrel{*}{\mathfrak{F}}^{\mu \nu}, \nu
$$

Consequently, we get

$$
\stackrel{*}{\mathfrak{J}}_{, \mu}^{\mu} \stackrel{\text { def. }}{=} \stackrel{\mathfrak{F}}{\mathfrak{F}}_{, \mu \nu} \equiv 0 .
$$

Also, we can introduce the quantities

$$
\begin{gathered}
\stackrel{*}{\mathfrak{J}}^{2} \stackrel{\text { def. }}{=} \stackrel{*}{\mathfrak{J}}^{\mu} \stackrel{*}{\mathfrak{J}} \\
\stackrel{*}{\mu}^{\mu} \stackrel{\text { def. }}{=} \frac{\stackrel{\sim}{\mathfrak{J}}^{\mu}}{\lambda}, \\
U^{\mu} \stackrel{\text { def. }}{=} \frac{J^{\mu}}{J},
\end{gathered}
$$

where $\stackrel{*}{J^{2}} \stackrel{\text { def. }}{=} J^{\mu} J_{\mu}^{*}$. Considering the relations given in the present section, an apparent comparison with EinsteinMaxwell's theory, we may attribute the following physical meaning to the geometric entities given above:

(1) equations (38) (or consequently (40)) as a generalization of Maxwell's dynamical equations,

(2) the vector $\stackrel{*}{c}_{\mu}$, as a generalization of electromagnetic potential of the electromagnetic field,

(3) the tensor $\stackrel{*}{F}_{\mu \nu}$, as a geometric representation of the electromagnetic field strength,

(4) the vector density given by (42) as a geometric representative of the current vector density,

(5) the scalar $\mathfrak{J}$, given by (44), as a geometric representative of the electromagnetic charge density,

(6) the identity (43) as representing conservation of the charge density.

In general, we can take (38) as being responsible for the electromagnetic field sector of the suggested theory. Justification of the above attributions is given in the next subsections.

4.2. Weak Field Approximation. In this subsection, we give the second method used to attribute physical meaning to the geometric objects admitted by the theory. This is applied by comparing the present theory with linear field theories. In this method we assume a weak static field and a slowly moving test particle. All the abovementioned physical meanings (given in Section 4.1) can be more justified by considering weak field approximation. Although the scheme of weak fields is, in general, not covariant, it gives physical information about the geometric content of any nonlinear field theory. Let us assume that the field variables $\lambda_{i}$ of the theory (the $\mathrm{BB}$ of the PAP-geometry) may be written as

$$
\lambda_{i}=\delta_{i \mu}+\epsilon h_{i}
$$

where $\delta_{i \mu}$ is Kronecker delta, $h_{i}$ are functions of the coordinates representing deviation of the PAP-space from flat space, and $\epsilon$ is a small dimensionless parameter whose square can be neglected (weak field approximation). Then using (1) and (47) we can expand $\lambda_{i}^{\mu}$ in the form

$$
\underset{i}{\lambda_{i}^{\mu}}=\delta_{i \mu}-\epsilon h_{i}^{\mu}+\epsilon^{2} h_{j}^{\mu} h_{i}^{j}-\cdots
$$

In what follows, we are concerned with the order of the parameter $\epsilon$, not $b$. Since the PAP-geometry has the same $\mathrm{BB} \lambda_{i}$ as AP-geometry, then all tensors of PAP will have the same order in $\epsilon$ as in the AP. The fundamental tensors in AP are expanded as given in [32]. Table 2 gives the order of magnitude of connections and tensors, of the space concerned, expanded in terms of the parameter $\epsilon$, where we write the general expansion form, of any geometric object $s$, as

$$
s=\epsilon^{0} s^{(0)}+\epsilon^{1} s^{(1)}+\epsilon^{2} s^{(2)}+\cdots+\epsilon^{r} s^{(r)},
$$

where $s^{(r)}$ is the $r$ th order of the term.

Table 2 displays different orders of magnitude, of the parameter $\epsilon$ for each geometric object. The sign $\checkmark$ indicates that a certain order exists in the expansion formula, while the sign $\otimes$ indicates the absence of this term.

Using Table 2, we can write the linearized form of the field equations (30) as follows.

4.2.1. The Linearized Symmetric Part of the Field Equations. This part may be written in the form

$$
R_{\mu \nu}^{(1)}-\frac{1}{2} \delta_{\mu \nu} R^{(1)}=\stackrel{*}{T}_{\mu \nu}^{(1)}
$$

or equivalently,

$$
R_{\mu \nu}^{(1)}=\stackrel{*}{T}_{\mu \nu}^{(1)}-\frac{1}{2} \delta_{\mu \nu}{ }^{*}{ }^{(1)},
$$

where $\stackrel{*}{T}^{(1)}$ is the trace of $\stackrel{*}{T}_{\mu \nu}^{(1)}$ and

$$
\stackrel{*}{T}_{\mu \nu}^{(1)} \stackrel{\text { def. }}{=}-\delta_{\mu \nu} \frac{b}{2} \stackrel{*}{\theta}^{(1)}+\frac{b}{2} \stackrel{*}{\theta}_{\mu \nu}^{(1)}-\frac{3}{4} b \stackrel{*}{\psi}_{\mu \nu}^{(1)} \text {. }
$$

Consequently,

$$
\begin{aligned}
\stackrel{*}{T}^{(1)} & =-2 b \stackrel{*}{\theta}^{(1)}+\frac{b}{2} \stackrel{*}{\theta}^{(1)}-\frac{3}{4} b \stackrel{*}{\psi}^{(1)} \\
& =-\frac{3}{2} b \stackrel{*}{\theta}^{(1)}-\frac{3}{4} b \stackrel{*}{\psi}^{(1)} \\
& =-\frac{3}{4} b \stackrel{*}{\theta}^{(1)}=\frac{3}{4} b \stackrel{*}{\psi}^{(1)}
\end{aligned}
$$


TABLE 2: Expansion of the geometric objects.

\begin{tabular}{|c|c|c|c|c|}
\hline Geometric objects & Terms of 0 -order & Terms of first order & $\begin{array}{l}\text { Order of } \epsilon \\
\text { Terms of second order }\end{array}$ & Terms of third and higher order \\
\hline$\lambda_{i} \mu$ & $\checkmark$ & $r$ & $\otimes$ & $\otimes$ \\
\hline$g_{\mu \nu}$ & $\checkmark$ & $\checkmark$ & $\checkmark$ & $\otimes$ \\
\hline$\lambda^{\mu}$ & $\checkmark$ & $\checkmark$ & $\checkmark$ & $\checkmark$ \\
\hline$g^{i \nu}$ & $\checkmark$ & $\checkmark$ & $\checkmark$ & $\checkmark$ \\
\hline$\lambda$ & $\checkmark$ & $\checkmark$ & $\checkmark$ & $\checkmark$ \\
\hline$g \stackrel{\text { def. }}{=}\left|g_{\mu \nu}\right|$ & $\checkmark$ & $\checkmark$ & $\checkmark$ & $\checkmark$ \\
\hline$\left\{\begin{array}{c}\alpha \\
\mu \nu\end{array}\right\}$ & $\otimes$ & $\checkmark$ & $\checkmark$ & $\checkmark$ \\
\hline$\gamma_{. \mu \nu}^{*}$ & $\otimes$ & $\checkmark$ & $\checkmark$ & $\checkmark$ \\
\hline$\nabla_{. \mu \nu}^{\alpha^{\mu \nu}}$ & $\otimes$ & $\checkmark$ & $\checkmark$ & $\checkmark$ \\
\hline$\stackrel{*}{\Lambda}_{\cdot \mu \nu}^{\alpha}$ & $\otimes$ & $\checkmark$ & $\checkmark$ & $\checkmark$ \\
\hline$\stackrel{*}{\Delta}_{\cdot \mu \nu}^{\alpha}$ & $\otimes$ & $\checkmark$ & $\checkmark$ & $\checkmark$ \\
\hline$\stackrel{*}{c_{\alpha}}$ & $\otimes$ & $\checkmark$ & $\checkmark$ & $\checkmark$ \\
\hline$\stackrel{*}{\xi}_{\mu \nu}$ & $\otimes$ & $\checkmark$ & $\checkmark$ & $\checkmark$ \\
\hline$\stackrel{*}{\chi}_{\mu \nu}$ & $\otimes$ & $\checkmark$ & $\checkmark$ & $r$ \\
\hline$\stackrel{*}{\varepsilon}_{\mu \nu}^{*}$ & $\otimes$ & $\checkmark$ & $\checkmark$ & $\checkmark$ \\
\hline$\zeta_{\mu \nu}^{*}$ & $\otimes$ & $\otimes$ & $\checkmark$ & $\checkmark$ \\
\hline$\stackrel{*}{\eta}_{\mu \nu}$ & $\otimes$ & $\otimes$ & $\checkmark$ & $\checkmark$ \\
\hline$\stackrel{*}{\kappa}_{\mu \nu}$ & $\otimes$ & $\otimes$ & $r$ & $\checkmark$ \\
\hline $\begin{array}{l}* \\
\theta_{\mu \nu}^{*} \\
*\end{array}$ & $\otimes$ & $\checkmark$ & $r$ & $\checkmark$ \\
\hline$\stackrel{*}{\psi}_{\mu \nu}$ & $\otimes$ & $\checkmark$ & $\checkmark$ & $\checkmark$ \\
\hline$\phi_{\mu \nu}$ & $\otimes$ & $\otimes$ & $\checkmark$ & $\checkmark$ \\
\hline$\stackrel{*}{\omega}_{\mu \nu}$ & $\otimes$ & $\otimes$ & $\checkmark$ & $\checkmark$ \\
\hline$\stackrel{*}{\omega}_{\mu \nu}$ & $\otimes$ & $\otimes$ & $\checkmark$ & $r$ \\
\hline$\stackrel{*}{\sigma}_{\mu \nu}$ & $\otimes$ & $\otimes$ & $\checkmark$ & $\checkmark$ \\
\hline$\stackrel{*}{\alpha}_{\mu \nu}$ & $\otimes$ & $\otimes$ & $\checkmark$ & $r$ \\
\hline
\end{tabular}

The right-hand side of the linearized symmetric equation, (51), may be written in the following form, using (52) and (53),

$$
\begin{aligned}
\stackrel{*}{(1)}_{\mu \nu}^{(1)} & -\frac{1}{2} \delta_{\mu \nu} \stackrel{*}{(1)}^{(1)} \\
& =-\frac{b}{2} \delta_{\mu \nu} \stackrel{*}{\theta}^{(1)}+\frac{b^{*}}{2} \theta^{(1)}{ }_{\mu \nu}-\frac{3}{4} b \stackrel{*}{\psi}_{\mu \nu}^{(1)}+\frac{3}{8} b \delta_{\mu \nu} \stackrel{*}{ }^{(1)} \\
& =-\frac{b}{8} \delta_{\mu \nu}{ }^{*} \theta^{(1)}+\frac{b}{2} \theta_{\mu \nu}^{* 1)}-\frac{3}{4} b \stackrel{*}{\psi}_{\mu \nu}^{(1)} .
\end{aligned}
$$

The left-hand side of the linearized symmetric field equations (51) can be written in the form

$$
R_{\mu \nu}^{(1)}=y_{\mu \nu, \alpha \alpha}=\nabla^{2} y_{\mu \nu}
$$

since the field is static $\alpha=1,2,3$, and $y_{\mu \nu}$ is defined as

$$
y_{\mu \nu} \stackrel{\text { def. }}{=} h_{\mu}+h_{\nu} \text {. }
$$

Using (54) and (55) we can write the linearized symmetric field equations (51) in the form

$$
\nabla^{2} y_{\mu \nu}=-\frac{b}{8} \delta_{\mu \nu} \stackrel{*}{\theta}^{(1)}+\frac{b}{2} \stackrel{*}{\theta}_{\mu \nu}^{(1)}-\frac{3}{4} b \stackrel{*}{\psi}_{\mu \nu}^{(1)} .
$$

As usually done, in order to compare the linear result (57) with Newton's theory, we examine the case $\mu=\nu=0$; then

$$
\nabla^{2} y_{00}=-\frac{b}{8} \stackrel{*}{\theta}^{(1)}-\frac{3}{4} b \stackrel{*}{\psi}_{00}^{(1)}
$$

since the field is static, $\stackrel{*}{(1)}_{00}=0$. Also, we have $\stackrel{*}{\theta}=-\stackrel{*}{*}$, so, we may write (58) as

$$
\nabla^{2} y_{00}=\frac{b}{8} \stackrel{*}{\psi}^{(1)}-\frac{3}{4} b \stackrel{*}{\psi}_{00}^{(1)}
$$

Now define the function

$$
\Phi \stackrel{\text { def. }}{=}-\frac{8}{a}\left(g_{00}-1\right),
$$

where $a$ is a parameter; then

$$
\nabla^{2} \Phi=-\frac{8}{a} \nabla^{2} y_{00} .
$$

Substituting from (61) into (59), we get

$$
\nabla^{2} \Phi=-\frac{b}{a}\left(\stackrel{*}{\psi}^{(1)}-6 \psi_{00}^{*(1)}\right) .
$$


The ultimate goal of linearizing any nonlinear field theory is to compare the theory with classical, partially successful linear field theory and also to attribute physical meaning to the geometric objects. In the present case the symmetric part of the field equations of the theory (62) is to be compared with Newton's theory, that is, with Poisson's equation. It seems from (62) that the tensor $\stackrel{*}{\psi}_{\mu \nu}$, in its linear form, is responsible for representing the material distribution. Let us make a one to one correspondence between the components of $\stackrel{*}{\psi}_{\mu \nu}^{(1)}$ and those $T_{\mu \nu}^{(1)}$ (the phenomenological matter tensor usually used in GR given in (35)). The spatial components of $T_{\mu \nu}^{(1)}$, for the isotropic perfect fluid, are attributed to the hydrostatic pressure [33], while the temporal component gives rise to the fluid density. Now, on one hand, pressure is associated with speed of the fluid particles and this is neglected since it would be of second order. On the other hand, classically speaking, pressure cannot produce gravity. So, from the one to one correspondence mentioned above and assuming that

$\stackrel{*}{\psi}_{\mu \nu}^{(1)}$ describe an isotropic perfect fluid, we can write

$$
\stackrel{*}{\psi}^{(1)}=\stackrel{*}{\psi}_{00}^{(1)} .
$$

Using this relation, then (62) can be written as

$$
\nabla^{2} \Phi=\frac{5 b}{a} \psi_{00}^{*}
$$

Equation (64) can be considered as the geometric form of "Poisson's equation" of Newton's theory with $\Phi$ representing the gravitational potential and $\stackrel{*}{\psi}_{\mu \nu}^{(1)}$ the material source generating this potential. This justifies the physical attributions given in Section 4.1 which are

(i) $g_{\mu \nu}$ as the gravitational potential,

(ii) $\stackrel{*}{T}_{\mu \nu}$ as the geometric representation of the material distribution.

Taking $b=0$, then (64) can be written in the form

$$
\nabla^{2} \Phi=0
$$

This is "Laplace's equation" giving the gravitational field in free space. This gives further justifications of the two attributions given above.

4.2.2. The Linearized Skew Part of the Field Equations. The linearized form of the skew part of the field equations (30) can be written as

$$
\stackrel{*}{F}_{\mu \nu}^{(1)}=-\frac{b}{2}\left(\stackrel{*}{c}_{\mu, \nu}^{(1)}-\stackrel{*}{c}_{\nu, \mu}^{(1)}\right),
$$

where

$$
\stackrel{*}{F}_{\mu \nu}^{(1)} \stackrel{\text { def. }}{=}-\frac{b}{4} \stackrel{*}{\chi}_{\mu \nu}^{(1)}+\frac{b^{*}}{2} \stackrel{\xi}{\mu \nu}^{(1)} .
$$

In the case of static electromagnetic field, the definition (42) gives

$$
\stackrel{*}{J}_{1}^{(1)}=\stackrel{*}{J}_{2}^{(1)}=\stackrel{*}{J}_{3}^{(1)}=0, \quad \stackrel{*}{J}_{0}^{(1)}=\stackrel{*}{F}_{0 v, v}^{(1)}
$$

and the field equations (66) give

$$
\stackrel{*}{F}_{0 v, v}^{(1)}=-\frac{b}{2} *_{0, v v}^{(1)}
$$

(note that $v=1,2,3$ since the field is static). Equation (69) can be written as

$$
\stackrel{*(1)}{F_{0 v, v}}=-\frac{b}{2} \nabla^{2} c_{0}^{(1)}
$$

or using (68), the above equation can be written as

$$
\nabla^{2} \stackrel{*}{c}_{0}^{(1)}=\stackrel{*}{J}_{0}
$$

Now Poisson's equation for an electric charge distribution with electric charge density $q$ and electric potential $\varphi$ can be written as

$$
\nabla^{2} \varphi=\frac{4 \pi q}{\varepsilon}
$$

where $\varepsilon$ is the dielectric constant. A comparison between (71) and (72) shows the following.

(i) Equation (71) can be considered as the geometric form of Poisson's equation.

(ii) $\stackrel{*}{c_{0}}(=\varphi)$ is the electric potential.

(iii) ${ }^{*} J_{0}(=4 \pi q / \varepsilon)$ is the geometric charge density.

(iv) Conservation of charge density is guaranteed via (43).

It is clear that if $q=0$, that is, $J_{0}^{*}=0,(71)$ reduces to the Laplace equation for the same problem

$$
\nabla^{2} c_{0}^{(1)}=0
$$

Also, the current vector density (42) will give, using (67),

$$
\stackrel{*}{\mathfrak{J}}_{\mu}^{(1)} \stackrel{\text { def. }}{=}\left(-\frac{b}{4} \chi_{\mu \nu}^{*(1)}+\frac{b^{*}{ }^{(1)}}{\xi_{\mu \nu}}\right)_{, \nu} .
$$

The above physical attributions support those given at the end of Section 4.1.

4.3. The Type of a Certain Geometric Structure. Each field theory has its domain of applicability. A geometric field theory is constructed in the framework of a space while applications of the theory are carried out in the context of geometric structures. For example, the field theory suggested in the present work is constructed in the context of the PAP-space. The study given in the above two subsections shows that the theory suggested represents a unified field which is split into gravity and electromagnetic fields especially in the linearized 
form of the theory. A new feature of the theory is that it gives a geometric description of a material distribution. To support these representations several applications are needed. Such applications are to be carried out using geometric structures as stated above. A scheme is needed to explore the capabilities of a certain geometric structure to represent physical entities, in the context of the field theory suggested. As a trivial example, in the context of GR, a geometric structure with vanishing curvature cannot be used to represent gravity. The idea has been generalized in a scheme called "type analysis." This scheme has been suggested by Mikhail and Wanas in 1981 [34] in the context of generalized field theory (GFT) [35]. This scheme is shown to be useful in applications (cf. [3640]). In general it is a covariant scheme for analyzing any specific geometric PAP-structure (with certain symmetries) in the context of the theory suggested. In other words it gives the capabilities of a certain structure to represent physical entities. In Table 3, we give the physical type of the BB of any AP-structure in the context of the theory derived in the present work. The scheme depends mainly on the orders of magnitude of some tensors as given in Table 2.

It is to be considered that since the $\mathrm{BB}$ of the AP and the PAP-geometry are the same, the generalization of the scheme of type analysis is not difficult. The vanishing of a certain tensor in the AP-space implies the vanishing of its parameterized version in the PAP-space.

In constructing Table 3, we use the following definitions:

$$
\begin{gathered}
\stackrel{*}{Z_{\mu \nu}} \stackrel{\stackrel{\text { def. }}{=}}{=} \frac{1}{4}(1-b) \stackrel{*}{\kappa}_{\mu \nu}-\frac{1}{4}(2-b) \stackrel{*}{\eta}_{\mu \nu}-\frac{1}{2} b \stackrel{*}{\zeta}_{\mu \nu}, \\
\stackrel{*}{M}_{\mu \nu} \stackrel{\text { def. }}{=} \frac{1}{2} \stackrel{*}{\sigma}_{\mu \nu}+\frac{1}{4}(1-3 b) \stackrel{*}{\omega}_{\mu \nu}-\frac{3}{2} b \stackrel{*}{\omega}_{\mu \nu}-\frac{1}{4}(2-5 b) \stackrel{*}{\phi}_{\mu \nu}, \\
\stackrel{* \text { def. }}{\Lambda}-\frac{1}{2}(1-2 b) \stackrel{*}{\alpha}-\frac{1}{4} \stackrel{*}{\sigma}+\frac{1}{4} \stackrel{*}{\omega},
\end{gathered}
$$

which lead to

$$
\begin{aligned}
& \stackrel{*}{T}_{\mu \nu} \stackrel{\text { def. }}{=} \stackrel{*}{M}_{\mu \nu}+g_{\mu \nu}\left(\stackrel{*}{\Lambda}-\frac{b}{2} \stackrel{*}{\theta}\right)+\frac{b}{2} \stackrel{*}{\theta}_{\mu \nu}-\frac{3}{4} b \stackrel{*}{\psi}_{\mu \nu}, \\
& \stackrel{*}{F}_{\mu \nu} \stackrel{\text { def. }}{=} \stackrel{*}{Z}_{\mu \nu}-\frac{b}{4} \stackrel{*}{\chi}_{\mu \nu}+\frac{b^{*}}{2} \stackrel{\xi}{\mu \nu}
\end{aligned}
$$

The type of a certain AP- (or PAP-) structure is written as a combination of the two groups of codes F, G, as will appear in the following section. Although the scheme of type analysis depends mainly on the expansion Table 2 (violating general covariance), this scheme is generally covariant. This is because it depends, as shown in Table 3, on the property of vanishing of some tensors. For example, the vanishing of Riemann-Christoffel curvature tensor $R_{. \beta \gamma \delta}^{\alpha}$ indicates that the space is flat and consequently has neither matter-energy nor fields. A weak field depends on the existence (nonvanishing) of tensors with linear terms in its expansion formula. A strong field depends on the nonvanishing of tensors, describing the field, whose expansion formula starts with second order quantities and so on. In the following section we give an example to illustrate the application of this scheme.

\section{The Case of Spherical Symmetry}

In what follows we give an example for applying the theory suggested and for exploring some of the advantages of the scheme of type analysis in the case of spherical symmetry. Also, this example illustrates the role of the parameter $(b)$ in this theory.

5.1. PAP Structure with Spherical Symmetry. Spherical symmetry represents the most simple case used to study many physical configurations. In 4-dimension, the most general AP-structure with spherical symmetry is that constructed by Robertson in 1932 [41], which, in spherical polar coordinates $\left(x^{0} \equiv t, x^{1} \equiv r, x^{2} \equiv \theta, x^{3} \equiv \phi\right)$, can be written in the following matrix:

$$
\lambda_{i}^{\mu}=\left(\begin{array}{cccc}
A & D r & 0 & 0 \\
0 & B \sin \theta \cos \phi & \frac{B}{r} \cos \theta \cos \phi & -\frac{B \sin \phi}{r \sin \theta} \\
0 & B \sin \theta \sin \phi & \frac{B}{r} \cos \theta \sin \phi & \frac{B \cos \phi}{r \sin \theta} \\
0 & B \cos \theta & -\frac{B}{r} \sin \theta & 0
\end{array}\right) \text {, }
$$

where $A, B$, and $D$ are unknown functions of $r$ only. Using relation (1), we can write the covariant components of (77) in the form

$$
\lambda_{i}=\left(\begin{array}{cccc}
\frac{1}{A} & 0 & 0 & 0 \\
-\frac{r D}{A B} \sin \theta \cos \phi & \frac{1}{B} \sin \theta \cos \phi & \frac{r}{B} \cos \theta \cos \phi & -\frac{r}{B} \sin \theta \sin \phi \\
-\frac{r D}{A B} \sin \theta \sin \phi & \frac{1}{B} \sin \theta \sin \phi & \frac{r}{B} \cos \theta \sin \phi & \frac{r}{B} \sin \theta \cos \phi \\
-\frac{r D}{A B} \cos \theta & \frac{1}{B} \cos \theta & -\frac{r}{B} \sin \theta & 0
\end{array}\right) .
$$


TABle 3: Type analysis.

\begin{tabular}{|c|c|c|}
\hline Tensor values & Physical meaning & Code \\
\hline$F_{\mu \nu}^{*}=0$ & No electromagnetic field & $F 0$ \\
\hline$\stackrel{*}{F}_{\mu \nu} \neq 0, \stackrel{*}{Z}_{\mu \nu}=0$ & $\begin{array}{c}\text { Weak electromagnetic field } \\
\text { (since } \stackrel{*}{Z}_{\mu \nu} \text { has no linear terms in } \epsilon \text { (see Table 2)) }\end{array}$ & $F I$ \\
\hline$\stackrel{*}{F}_{\mu \nu} \neq 0, \stackrel{*}{Z}_{\mu \nu} \neq 0$ & Strong electromagnetic field & FII \\
\hline$R_{. \beta \gamma \delta}^{\alpha}=0, \stackrel{*}{Q}_{\beta \gamma \delta}^{\alpha}=0$ & No gravitational field & G0 \\
\hline$R_{. \beta \gamma \delta}^{\alpha} \neq 0, \stackrel{*}{T}_{\mu \nu}=0, \stackrel{*}{M}_{\mu \nu}=0, \stackrel{*}{\Lambda}=0$ & Gravitational field in free space & GI \\
\hline$R_{. \beta \gamma \delta}^{\alpha} \neq 0, \stackrel{*}{T} \mu \nu, \stackrel{*}{M} \mu, \stackrel{*}{\Lambda}=0$ & $\begin{array}{l}\text { Gravitational field within a material distribution } \\
\text { (since } \stackrel{*}{M}_{\mu \nu} \text { does not contain any linear terms in } \epsilon \text { ) }\end{array}$ & GII \\
\hline$R_{. \beta \gamma \delta}^{\alpha} \neq 0, \stackrel{*}{M_{\mu \nu}} \neq 0, \stackrel{*}{\Lambda}=0$ & Strong gravitational field within a material distribution & GIII \\
\hline$R_{. \beta \gamma \delta}^{\alpha} \neq 0, \stackrel{*}{M}_{\mu \nu} \neq 0, \stackrel{*}{\Lambda} \neq 0$ & Very strong gravitational field within a material distribution & GIV \\
\hline
\end{tabular}

The Riemannian space defined from the PAP-structure (78) is given, using (3), by

$$
\begin{aligned}
d s^{2}= & \frac{B^{2}+D^{2} r^{2}}{A^{2} B^{2}} d t^{2}-\frac{2 D r}{A B^{2}} d t d r \\
& +\frac{1}{B^{2}}\left(d r^{2}+r^{2} d \theta^{2}+r^{2} \sin ^{2} \theta d \phi^{2}\right) .
\end{aligned}
$$

Let us first apply the scheme of type analysis, given in Section 4.3, to the tetrad (77). To get the type of this tetrad, we have to calculate the tensors listed in Table 3. In these calculations, we have used the algebraic manipulation language REDUCE 3.6. From definitions (75) and (76), we get the following nonvanishing components of the tensors necessary to get the type of the structure (77):

$$
\begin{aligned}
& \stackrel{*}{F}_{\mu \nu} \neq 0, \quad \stackrel{*}{Z}_{\mu \nu} \neq 0, \\
& \stackrel{*}{T}_{\mu \nu} \neq 0, \quad \stackrel{*}{M_{\mu \nu}} \neq 0, \quad \stackrel{*}{\Lambda} \neq 0 .
\end{aligned}
$$

Also, in addition to these values we get $R_{. \mu \nu \sigma}^{\alpha} \neq 0,{ }_{Q}^{*}{ }_{. \mu \nu \sigma}^{\alpha} \neq 0$. The values of the nonvanishing components of these tensors are not listed here, since these are too long.

Consequently, using Table 3 and the above results, the type of tetrad (77) in the context of present field theory is FIIGIV; that is, the $\mathrm{BB}$ (77) are capable of describing a unified strong electromagnetic and gravitational field within a material distribution.

In the present section, we aim to give an example, as simple as possible, to support the following points:

(i) the physical meaning attributed to some geometric object given in Sections 4.1 and 4.2,

(ii) the role of the scheme of type analysis in simplifying the problem,

(iii) the present theory implying all the advantages of GR in similar cases.
Further simplification will make the above points more clear. This is done in the following two subsections.

5.2. The Case of Pure Gravity. For simplicity, we consider pure gravity in the present subsection. If we take $\mathbf{D}=\mathbf{0}$ the type becomes FOGIV, because of the vanishing of all skew tensors of Table 1, identically, which leads to

$$
\stackrel{*}{F}_{\mu \nu}=0, \quad \stackrel{*}{Z}_{\mu \nu}=0,
$$

while

$$
\begin{gathered}
R_{\mu \nu \sigma}^{\alpha} \neq 0, \quad \stackrel{*}{Q}_{. \mu \nu \sigma}^{\alpha} \neq 0, \quad{ }^{*} T_{\mu \nu} \neq 0, \\
\stackrel{*}{M}_{\mu \nu} \neq 0, \quad \stackrel{*}{\Lambda} \neq 0 .
\end{gathered}
$$

This means that, with $D=0$, the $\mathrm{BB}$ (77) can be used to study a strong gravitational field within material distribution. This shows the importance of type analysis. It represents a way for gaining some physical meaning of the geometric objects of the field equations before solving such equations. After taking $D=0$ (i.e. type F0GIV), the solution of the field equations will give rise to interior gravitational field, free from electromagnetism. The tetrad vector field (77) for the case $D=0$ may be written in the form

$$
\lambda_{i}^{\mu}=\left(\begin{array}{cccc}
A & 0 & 0 & 0 \\
0 & B \sin \theta \cos \phi & \frac{B}{r} \cos \theta \cos \phi & -\frac{B \sin \phi}{r \sin \theta} \\
0 & B \sin \theta \sin \phi & \frac{B}{r} \cos \theta \sin \phi & \frac{B \cos \phi}{r \sin \theta} \\
0 & B \cos \theta & -\frac{B}{r} \sin \theta & 0
\end{array}\right),
$$


and also

$$
\lambda_{i}=\left(\begin{array}{cccc}
\frac{1}{A} & 0 & 0 & 0 \\
0 & \frac{1}{B} \sin \theta \cos \phi & \frac{r}{B} \cos \theta \cos \phi & -\frac{r}{B} \sin \theta \sin \phi \\
0 & \frac{1}{B} \sin \theta \sin \phi & \frac{r}{B} \cos \theta \sin \phi & \frac{r}{B} \sin \theta \cos \phi \\
0 & \frac{1}{B} \cos \theta & -\frac{r}{B} \sin \theta & 0
\end{array}\right) .
$$

The metric of the Riemannian space defined using the PAPspace (84) may be written, using (3), as

$$
d s^{2}=\frac{1}{A^{2}} d t^{2}+\frac{1}{B^{2}}\left(d r^{2}+r^{2} d \theta^{2}+r^{2} \sin ^{2} \theta d \phi^{2}\right) .
$$

According to tetrad vector field (84) and using tensors definition in Table 1, the field equations (30) will give rise to the following set of differential equations in the unknown functions $A(r), B(r)$ :

$$
\begin{gathered}
K_{00}: \frac{1}{4 A^{4} r}\left(8 A^{2} B B^{\prime \prime} r b^{2}+8 A^{2} B B^{\prime \prime} r+16 A^{2} B B^{\prime} b^{2}\right. \\
+16 A^{2} B B^{\prime}-14 A^{2} B^{\prime 2} r b^{2}-12 A^{2} B^{2} r \\
-2 A B^{2} A^{\prime \prime} r b^{2}-4 A B^{2} A^{\prime} b^{2}+2 A B A^{\prime} B^{\prime} r b^{2} \\
\left.+3 B^{2} A^{\prime 2} r b^{2}\right)=0, \\
K_{11}: \frac{1}{4 A^{2} B^{2} r}\left(4 A^{2} B B^{\prime} b^{2}+8 A^{2} B B^{\prime}-2 A^{2} B^{\prime 2} r b^{2}\right. \\
-4 A^{2} B^{\prime 2} r+8 A B^{2} A^{\prime} b^{2}+8 A B^{2} A^{\prime} \\
\left.-8 A B A^{\prime} B^{\prime} r b^{2}-8 A B A^{\prime} B^{\prime} r+B^{2} A^{\prime 2} r b^{2}\right)=0, \\
k_{22}: \frac{r}{4 A^{2} B^{2}}\left(2 A^{2} B B^{\prime \prime} r b^{2}+4 A^{2} B B^{\prime \prime} r+2 A^{2} B B^{\prime} b^{2}\right. \\
+4 A^{2} B B^{\prime}-2 A^{2} B^{\prime 2} r b^{2}-4 A^{2} B^{\prime 2} r \\
+4 A B^{2} A^{\prime \prime} r b^{2}+4 A B^{2} A^{\prime \prime} r+4 A B^{2} A^{\prime} b^{2} \\
+4 A B^{2} A^{\prime}+2 A B A^{\prime} B^{\prime} r b^{2}-9 B^{2} A^{\prime 2} r b^{2} \\
\left.-8 B^{2} A^{\prime 2} r\right)=0, \\
k_{33}=\sin ^{2} \theta k_{22}=0 .
\end{gathered}
$$

The set (86) represents the differential equations governing the gravitational field within a material distribution as indicated by the type of structure (83). We start to study the more simple case by converting the structure (83) from F0GIV to FOGI which represents a gravitational field outside the material distribution. This is done in order to compare with similar results of GR. The most famous and successful solutions of the field equations of GR are the solutions in free space. So, we are looking for such solutions for the field equations (30) in free space. For this reason, the effect of $T_{\mu \nu}^{*}$ should be switched off. This is done in Section 5.3.

5.3. The Exterior Solution. We discuss the solution of the field equations (30) outside spherical material distribution. The condition is to switch off the effect of the tensor $\stackrel{*}{T}_{\mu \nu}$ (responsible for matter distribution). This can be achieved by taking $\mathbf{b}=\mathbf{0}$. This means that the type of the space (77), with $b=0$, becomes FOGI. This point will be discussed in Section 6 . The case corresponds to a pure gravitational field, with spherical symmetry, outside the material distribution. As a consequence, we get

$$
\begin{gathered}
R_{. \beta \gamma \delta}^{\alpha} \neq 0, \quad \stackrel{*}{Q}_{. \mu \nu \sigma}^{\alpha}=0, \quad \stackrel{*}{F}_{\mu \nu}=0, \\
\stackrel{*}{Z}_{\mu \nu}=0, \quad \stackrel{*}{T}_{\mu \nu}=0, \quad \stackrel{*}{M}_{\mu \nu}=0, \quad \stackrel{*}{\Lambda}=0 .
\end{gathered}
$$

So, the set of differential equations (86) will be reduced to

$$
\begin{gathered}
K_{00}: 2 \frac{B^{\prime \prime}}{B}+4 \frac{B^{\prime}}{B r}-3 \frac{B^{\prime 2}}{B^{2}}=0, \\
K_{11}: 2 \frac{B^{\prime}}{B r}-\frac{B^{\prime 2}}{B^{2}}+2 \frac{A^{\prime}}{A r}-2 \frac{A^{\prime} B^{\prime}}{A B}=0, \\
K_{22}: \frac{B^{\prime \prime}}{B}+\frac{B^{\prime}}{B r}-\frac{B^{\prime 2}}{B^{2}}+\frac{A^{\prime \prime}}{A}+\frac{A^{\prime}}{A r}-\frac{A^{\prime 2}}{A^{2}}=0, \\
K_{33}=\sin ^{2} \theta K_{22}=0 .
\end{gathered}
$$

The first equation in the set (88) is purely made of $B(r)$, which can be integrated to give

$$
B^{\prime}=c_{1} r^{-2} B^{3 / 2}
$$

Integrating again, we get

$$
B=\left(c_{2}+\frac{c_{1}}{2 r}\right)^{-2}
$$

where $c_{1}$ and $c_{2}$ are constant of integration. Substituting from (90) in the second equation of the set (88), we obtain, after integration,

$$
A=c_{3} \frac{\left(c_{2}+\left(c_{1} / 2 r\right)\right)}{\left(c_{2}-\left(c_{1} / 2 r\right)\right)},
$$

where $c_{3}$ is a third constant of integration. The functions $A(r)$ and $B(r)$ given by (90) and (91), respectively, satisfy the third equation of the set (88) without any further condition. Now, in order to get some physical meaning for the solution given by (90) and (91) and to compare with the results of GR, let us evaluate the metric of the pseudo-Riemannian space obtained from the tetrad 73

$$
g_{\mu \nu} \stackrel{\text { def }}{=} e_{i} \lambda_{i} \lambda_{i} \lambda_{\nu}
$$


where

$$
e_{i}=(+1,-1,-1,-1)
$$

is the Levi-Civita indicator. Substituting the solutions (90) and (91) into the above expression, we get

$$
\begin{aligned}
d \tau^{2}= & c_{3}^{2} \frac{\left(c_{2}-\left(c_{1} / 2 r\right)\right)^{2}}{\left(c_{2}+\left(c_{1} / 2 r\right)\right)^{2}} d t^{2}-\left(c_{2}+\frac{c_{1}}{2 r}\right)^{4} \\
& \times\left(d r^{2}+r^{2} d \theta^{2}+r^{2} \sin ^{2} \theta d \phi^{2}\right),
\end{aligned}
$$

where $\tau$ is the proper time. Taking

$$
c_{1}=m, \quad c_{2}=1=c_{3}
$$

we get

$$
\begin{aligned}
d \tau^{2}= & \frac{(1-(m / 2 r))^{2}}{(1+(m / 2 r))^{2}} d t^{2}-\left(1+\frac{m}{2 r}\right)^{4} \\
& \times\left(d r^{2}+r^{2} d \theta^{2}+r^{2} \sin ^{2} \theta d \phi^{2}\right) .
\end{aligned}
$$

This metric is identical to the Schwarzschild exterior field, in isotropic coordinates. It describes a pure gravitational field outside a spherically symmetric source having the geometric mass $(m)$. This will be discussed in the next section.

\section{Discussion and Concluding Remarks}

(1) In the present work, we constructed a field theory in the context of a PAP-space, a space with curvature and anticurvature, using the symmetric parameterized linear connection (9). From the analysis of the field equations of the theory, Section 4, it is shown that, in general, the theory is capable of representing gravity, antigravity, electromagnetism, and material distribution. The theory has the following general features.

(i) It is in general a covariant theory.

(ii) It is a pure geometric theory in the sense that all geometric objects that have been attributed to physics are constructed from the BB of the PAPgeometry.

(iii) The theory contains 16-field equations (30) and four equations of motion (21) which reduce to the field equations of GR and the ordinary geodesic equation, respectively, in the case $b=$ 0 . Consequently, all advantages of GR can be covered by the present theory under similar conditions. In view of the equations of motion, the theory violates the weak equivalence principle unless $b=0$. This case has been studied in Section 5.3.

(iv) Although the theory is, in general, not invariant under gauge transformation, its electromagnetic sector is gauge invariant. This is clear from the skew part (38) and its linearized form (66).
(2) The spherically symmetric solution obtained in Section 5.3 is a unique solution. It gives the Schwarzschild exterior field. This solution corresponds to $b=0$, as shown.

(3) The theory can be considered as a probe that explores the capabilities of a space to represent physics. From the analysis of the quantities of the theory given in Section 4, we can conclude the following.

(i) In the case $b=0$, the Riemannian case, the physics described contains the gravitational field in free space together with the background space-time.

(ii) In the case $b \neq 0$, the PAP-case, the physics that can be described includes gravity, antigravity, electromagnetism, and material distribution with possible mutual interactions.

(4) New physics may be discovered either by observations and experiment or by predictions of a certain theory. For example, on one hand, the results of SN type Ia observations may imply new physics. On the other hand, the definition of the anticurvature and the path equations, in the PAP-space, predict the existence of antigravity and a repulsive long range force, respectively. These predictions may interpret successfully SN type Ia observation [18]. This may be good motivation to construct field theories in spaces with curvature and anticurvature. The theory constructed and applied in the present work belongs to this type of theories.

(5) More applications of the present theory would give rise to new physics. This can be achieved by examining the following pionts.

(i) Solution of the differential equations (86) for which the function $D=0$ and $b \neq 0$ : the PAP-structure in this case has the type F0GIV. This means that we study a pure gravitational field within a material distribution. This may represent a further step for studying stellar interiors using a pure geometric approach [45].

(ii) Solution of the general equations with $D \neq 0$ and $b \neq 0$ : this corresponds to a PAP-structure of type FIIGIV. In general, the expected solution, in this case, would describe a strong unified gravitational and electromagnetic field within a material distribution.

(iii) Cosmological applications may solve, or at least throw some light on, some of the problems of standard cosmology.

(6) It is interesting to explore the role of the parameter $b$ and its relation to type analysis and consequently the applications of field theories in general. Figure 2 throws more light on the role of the parameter $b$ on the type of the structure used and possible physical applications. 
TABLE 4: Comparison between nonlinear geometric field theories $(n=4)$.

\begin{tabular}{|c|c|c|c|c|c|c|}
\hline Theory & Geometry & Number of field variables & Material energy tensor & $\begin{array}{l}\text { Metricity } \\
\text { condition }\end{array}$ & $\begin{array}{l}\text { Unification } \\
\text { philosophy }\end{array}$ & Anticurvature \\
\hline Einstein-Cartan [42] & Riemann-Cartan & $16+24$ & Phenomenological & Satisfied & Violated & Not defined \\
\hline Metric-Affine $[43,44]$ & Metric-Affine & $10+16+64$ & Phenomenological & Violated & Violated & Not defined \\
\hline Present Work & PAP & 16 & Geometric & Satisfied & Satisfied & Clearly defined \\
\hline
\end{tabular}

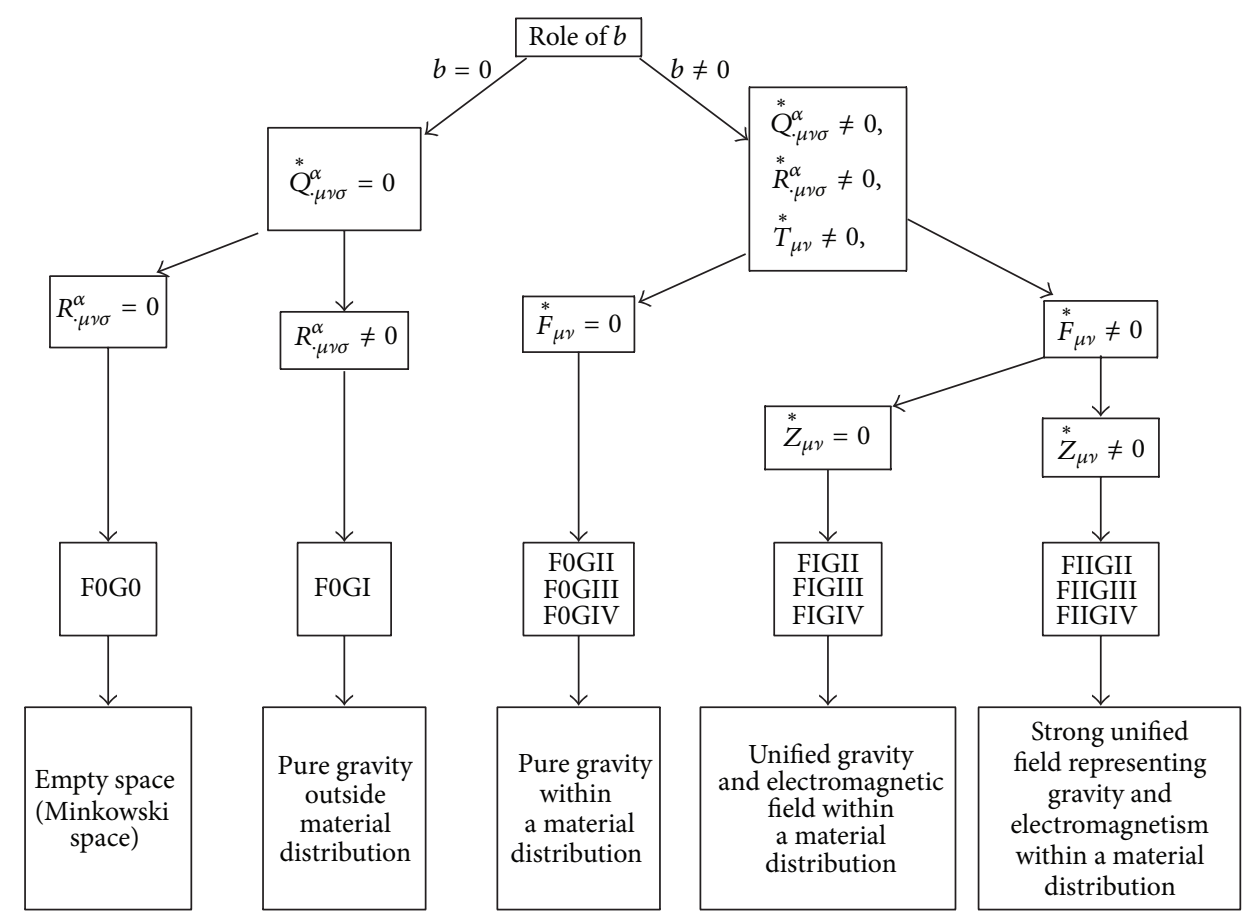

FIGURE 2: The role of the parameter $b$.

(7) The role of the anticurvature may be more illustrated in more sophisticated applications with $b \neq 0$ (see Figure 2). Such applications are now in progress.

(8) It is to be noted that in the present theory electromagnetism, material distribution, and gravity are nonminimally coupled.

(9) There are many theories, in the literature, with nonvanishing curvature and torsion. None of these theories has anticurvature or geometric material distribution. The anticurvature (17) is a new defined object which gives rise to antigravity ([18]). A theory including this object will lead to an interpretation of the observed accelerating expansion of the Universe. Another important point is that, in the context of geometrization, the unification philosophy implies that all fields and matter are generated from the same geometric entity. This philosophy is satisfied in the present work, in which gravity, electromagnetism, and material distribution are generated from the tetrad field (the BB of the geometry). Other theories violate this philosophy. However, Table 4 gives a brief comparison between some nonlinear geometric theories with curvature and torsion.

\section{Conflict of Interests}

The authors declare that there is no conflict of interests regarding the publication of this paper.

\section{References}

[1] J. L. Tonry, B. P. Schmidt, B. Barris et al., "Cosmological results from high-z supernovae," Astrophysical Journal, vol. 594, pp. 124, 2003.

[2] W. Hu and S. Dodelson, "Cosmic microwave background anisotropies," Annual Review of Astronomy and Astrophysics, vol. 40, pp. 171-216, 2002.

[3] Y. Sofue and V. Rubin, "Rotation curves of spiral galaxies," Annual Review of Astronomy and Astrophysics, vol. 39, pp. 137$174,2001$.

[4] S. M. Carroll, “The cosmological constant", Living Reviews in Relativity, vol. 4, p. 1, 2001.

[5] G. J. Olmo, "Limit to general relativity in $f(R)$ theories of gravity," Physical Review D: Particles, Fields, Gravitation, and Cosmology, vol. 75, no. 2, Article ID 023511, 2007.

[6] A. de Felice and S. Tsujikawa, " $\mathrm{f}(\mathrm{R})$ theories," Living Reviews in Relativity, vol. 13, no. 3, 2010.

[7] T. P. Sotiriou and V. Faraoni, " $f(R)$ theories of gravity," Reviews of Modern Physics, vol. 82, no. 1, pp. 451-497, 2010. 
[8] Y. Zhanga, H. Lic, Y. Gongb, and Z. Zhua, "Notes on $f(T)$ theories," Journal of Cosmology and Astroparticle Physics, vol. 7, article 015, 2011.

[9] G. G. L. Nashed, "A special exact spherically symmetric solution in $\mathrm{f}(\mathrm{T})$ gravity theories," General Relativity and Gravitation, vol. 45, no. 10, pp. 1887-1899, 2013.

[10] G. G. L. Nashed, "Spherically symmetric charged dS solution in $\mathrm{f}(\mathrm{T})$ gravity theory," Physical Review D, vol. 88, Article ID 104034, 2013.

[11] G. G. L. Nashed, "Schwarzschild solution in extended teleparallel gravity," Europhysics Letters, vol. 105, no. 1, article 10001, 2014.

[12] G. G. L. Nashed, "Local Lorentz transformation and exact spherically symmetric vacuum solutions in $f(T)$ gravity theories," The European Physical Journal C, vol. 73, Article ID 2394, 2014.

[13] K. Atazadeh and M. Mousavi, "Vacuum spherically symmetric solutions in $f(T)$ gravity," The European Physical Journal C, vol. 73, article 2272, 2013.

[14] M. I. Wanas, "Motion of spinning particles in gravitational fields," Astrophysics and Space Science, vol. 258, no. 1-2, pp. 237248, 1998.

[15] M. I. Wanas, "Parameterized absolute parallelism: a geometry for physical applications," Turkish Journal of Physics, vol. 24, pp. 473-488, 2000.

[16] M. I. Wanas, "Absolute parallelism geometry: developments, applications and problems," Studii şi Cercetări Ştiinţifice: Matematică University Bacău, no. 10, p. 297, 2001.

[17] M. I. Wanas, "The geometric origin of dark energy," AIP Conference Proceedings, vol. 1115, pp. 218-223, 2009.

[18] M. I. Wanas, "The other side of gravity and geometry: antigravity and anticurvature," Advances in High Energy Physics, vol. 2012, Article ID 752613, 10 pages, 2012.

[19] M. I. Wanas, M. Melek, and M. E. Kahil, "Quantum interference of thermal neutrons and spin-torsion interaction," Gravitation and Cosmology, vol. 6, pp. 319-322, 2000.

[20] A. A. Sousa and J. M. Maluf, "Gravitomagnetic effect and spintorsion coupling," General Relativity and Gravitation, vol. 36, no. 5, pp. 967-982, 2004.

[21] M. I. Wanas, M. Melek, and M. E. Kahil, "SN1987A: temporal models," in Proceedings of 9th MG Conference, vol. 2, p. 1100, World Scientific, 2002.

[22] F. I. Mikhail, "Tetrad vector fields and generalizing the theory of relativity," Ain Shams Science Bulletin, vol. 6, pp. 87-111, 1962.

[23] N. L. Youssef and A. M. Sid-Ahmed, "Extended absolute parallelism geometry," International Journal of Geometric Methods in Modern Physics, vol. 5, no. 7, pp. 1109-1135, 2008.

[24] N. L. Youssef and W. A. Elsayed, "A global approach to absolute parallelism geometry," Reports on Mathematical Physics, vol. 72, no. 1, pp. 1-23, 2013.

[25] M. M. Kamal, Spin-Torsion Interaction and Gravitomagnetism [M.Sc. Thesis], Ain Shams University, 2011.

[26] M. I. Wanas, "Dark energy: a missing physical ingredient," in Proceedings of the 6th Conference on Nuclear and Particle Physics, Luxor, Egypt, 2007, http://arxiv.org/abs/0809.5040.

[27] R. Colella, A. W. Overhauser, and S. A. Werner, "Observation of gravitationally induced quantum interference," Physical Review Letters, vol. 34, article 1472, 1975.

[28] J. L. Staudenmann, S. A. Werner, R. Colella, and A. W. Overhauser, "Gravity and inertia in quantum mechanics," Physical Review A, vol. 21, no. 5, pp. 1419-1438, 1980.
[29] S. A. Wener, H. Kaiser, M. Arif, and R. Clothier, "Neutron interference induced by gravity: new results and interpretations," Physica B, vol. 151, pp. 22-35, 1988.

[30] M. I. Wanas and M. E. Kahil, "Quantum features of nonsymmetric geometries," General Relativity and Gravitation, vol. 31, no. 12, pp. 1921-1929, 1999.

[31] M. I. Wanas, "From geometry to quantum," Algebras, Groups and Geometries, vol. 20, p. 345a, 2003.

[32] M. I. Wanas, A generalized field theory and its applications in cosmology [Ph. D. thesis], Cairo University, Giza, Egypt, 1975.

[33] R. Adler, M. Bazin, and M. Schiffer, Introduction to General Relativity, McGraw-Hill, New York, NY, USA, 2nd edition, 1975.

[34] F. I. Mikhail and M. I. Wanas, "A generalized field theory. II. Linearized field equations," International Journal of Theoretical Physics, vol. 20, no. 9, pp. 671-680, 1981.

[35] F. I. Mikhail and M. I. Wanas, "A generalized field theory. I. Field equations," Proceedings of the Royal Society of London A, vol. 356, no. 1687, pp. 471-481, 1977.

[36] M. I. Wanas, "A generalized field theory: charged spherical symmetric solution," International Journal of Theoretical Physics, vol. 24, no. 6, pp. 639-651, 1985.

[37] M. I. Wanas, "A self-consistent world model," Astrophysics and Space Science, vol. 154, no. 2, pp. 165-177, 1989.

[38] M. I. Wanas, "On the relation between mass and charge: a pure geometric approach," International Journal of Geometric Methods in Modern Physics, vol. 4, no. 3, pp. 373-388, 2007.

[39] R. S. de Souza and R. Opher, "Origin of $10^{15}-10^{16}$ G magnetic fields in the central engine of gamma ray bursts," Journal of Cosmology and Astroparticle Physics, vol. 2, article 022, 2010.

[40] R. S. de Souza and R. Opher, "Origin of intense magnetic fields near black holes due to non-minimal gravitationalelectromagnetic coupling," Physics Letters B, vol. 705, no. 4, pp. 292-293, 2011.

[41] H. P. Robertson, "Groups of motions in spaces admitting absolute parallelism," Annals of Mathematics, vol. 33, pp. 496520, 1932.

[42] F. W. Hehl, P. von der Heyde, G. D. Kerlick, and J. M. Nester, "General relativity with spin and torsion: foundations and prospect," Reviews of Modern Physics, vol. 48, no. 3, pp. 393-416, 1976.

[43] F. W. Hehl, J. D. McCreab, E. W. Mielkea, and Y. Ne'emanc, "Metric-affine gauge theory of gravity: field equations, noether identities, world spinors, and breaking of dilation invariance," Physics Reports, vol. 258, no. 1-2, pp. 1-171, 1995.

[44] F. W. Hehl, "Gauge theory of gravity and spacetime," Einstein Studies. In press.

[45] M. I. Wanas and S. A. Ammar, "A pure geometric approach to stellar structure," Central European Journal of Physics, vol. 11, no. 7, pp. 936-948, 2013. 

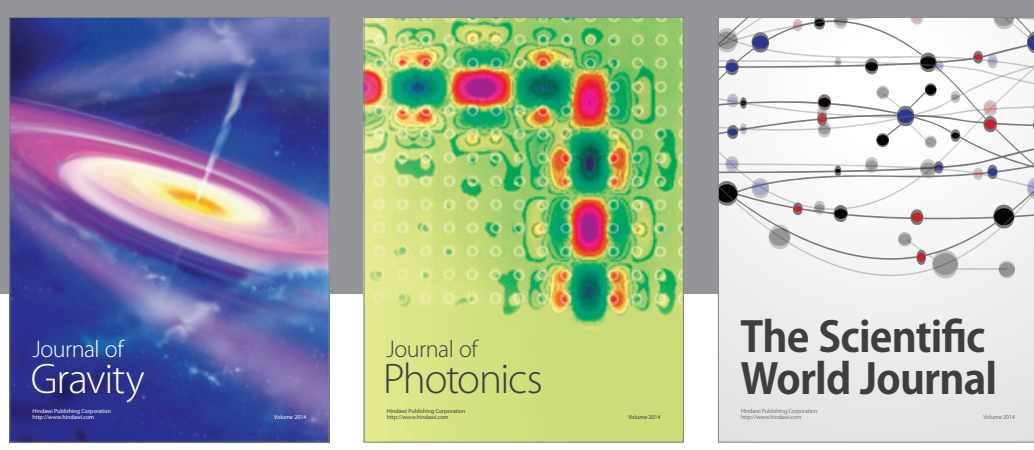

The Scientific World Journal
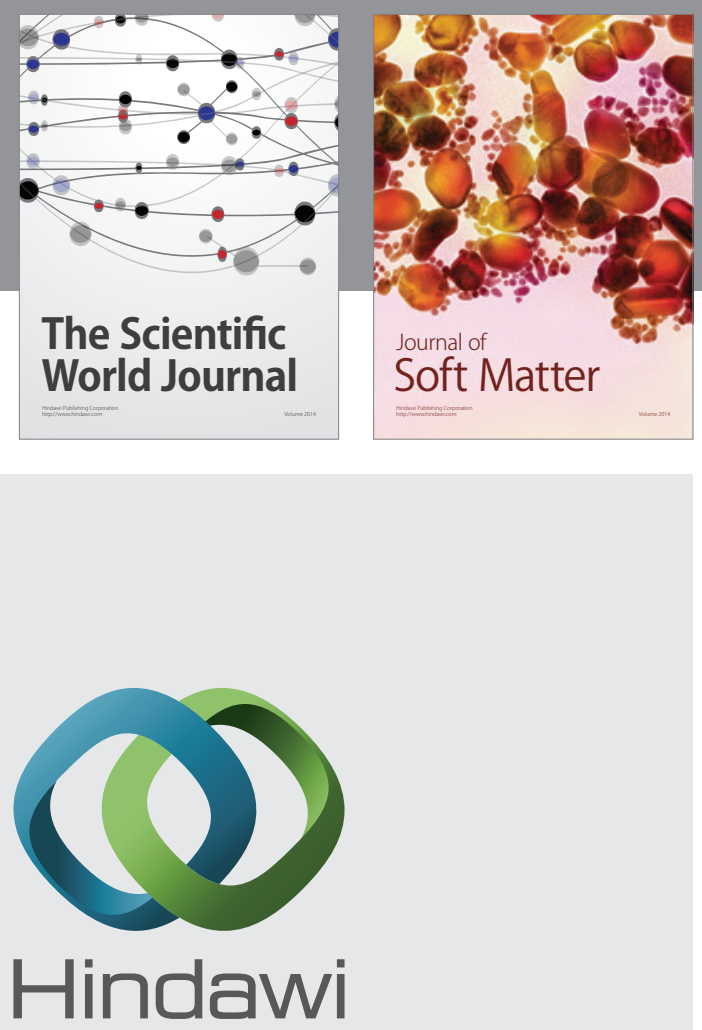

Submit your manuscripts at

http://www.hindawi.com

nternational Journal of

Statistical Mechanics
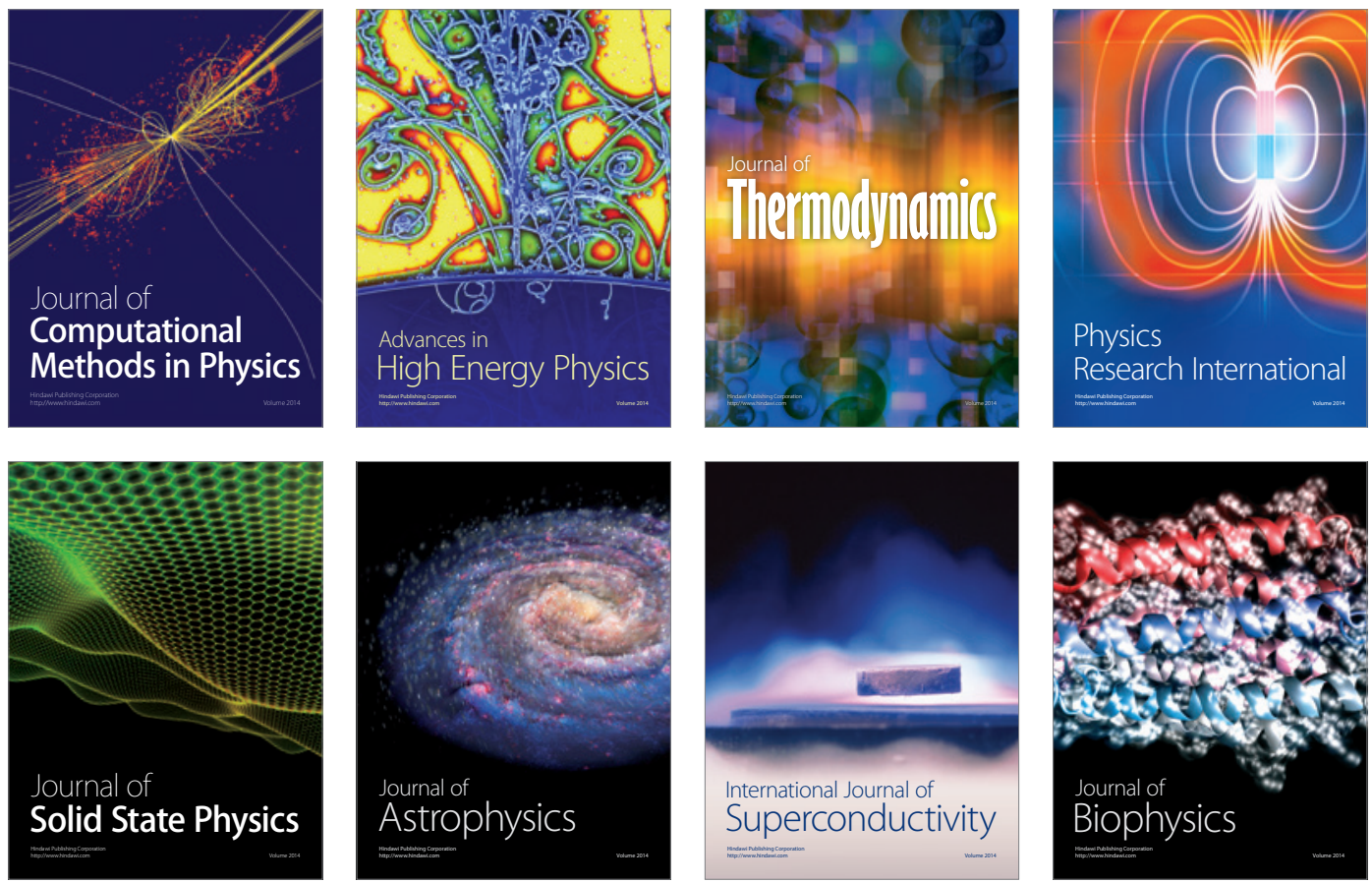
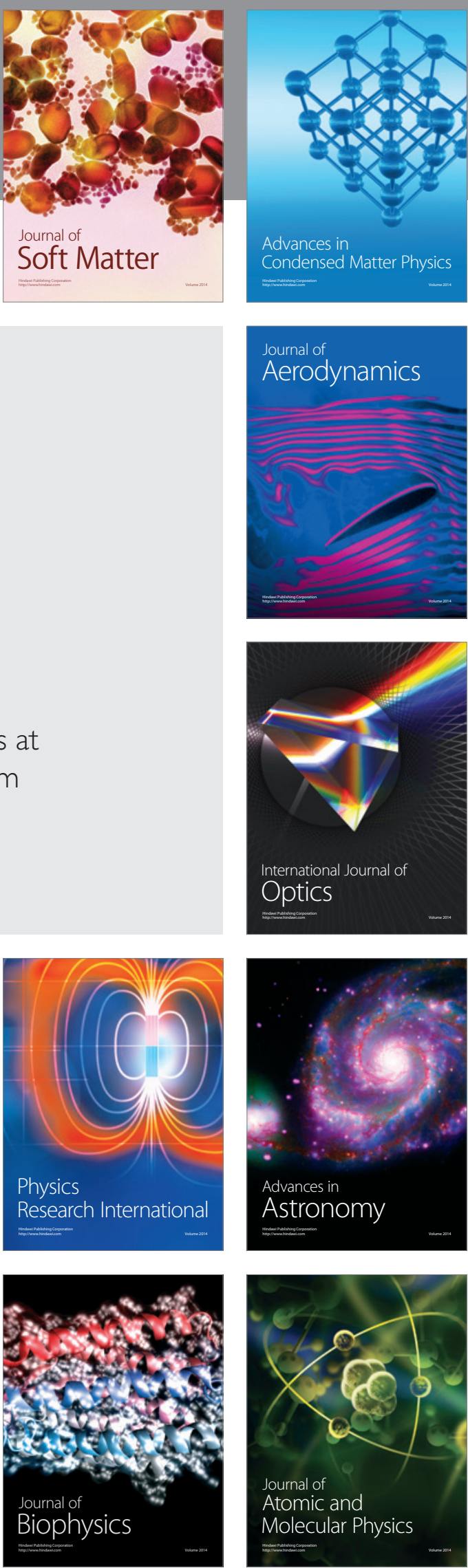